\title{
Guías 2009 de la Sociedad Chilena de Cardiología para el tratamiento del Infarto Agudo del Miocardio con supradesnivel del ST
}

\author{
Estas pautas fueron elaboradas por una comisión ad hoc nombrada por la Sociedad \\ Chilena de Cardiología, y estuvo constituida por: \\ Eduardo Guarda S1, Juan Carlos Prieto D2, Patricio Sanhueza C ${ }^{3}$, Christian \\ Dauvergne $\mathbf{M}^{4}$, René Asenjo G², Ramón Corbalán $\mathbf{H}^{1}$. \\ 1 Pontificia Universidad Católica de Chile \\ 2 Universidad de Chile \\ 3 Hospital San Juan de Dios \\ 4 Instituto Nacional del Tórax \\ Recibido el 1 de abril de 2009. Aceptado el 7 de julio de 2009
}

\section{Preámbulo}

Rev Chil Cardiol 2009; 28: 223-254

La Sociedad Chilena de Cardiología consideró necesaria una revisión de las Guías de manejo de Infarto Agudo del Miocardio (IAM), cuya primera versión apareció en 1997, y que fueron reeditadas en el 2002 $y$ en el 2004. Las nuevas recomendaciones que aparecen en esta versión se refieren especialmente a la necesidad de un diagnóstico más precoz, de optimizar la terapia de reperfusión mediante angioplastía primaria, al uso de desfibriladores implantables post IAM y a la evaluación previa al egreso hospitalario.

Para la confección de estas pautas esta comisión revisó la evidencia de la literatura y las pautas de distintos países; se eligió una redacción simple, de modo que puedan ser utilizadas rápidamente por médicos no especialistas. Se evitaron las categorizaciones según niveles de evidencia, las cuales el lector interesado puede buscarlas en la bibliografía al final de este documento1-7.

EI IAM constituye la causa directa de muerte de aproximadamente un $8 \%$ del total de fallecidos en Chile. Alrededor de un $30 \%$ de los pacientes con IAM fallecen antes de recibir atención médica. Cuando se han ingresado, la mortalidad promedio a nivel mundial es de aproximadamente un $10 \%$, cifra que oscila entre un 5 -8 \% en aquellos que reciben terapia de reperfusión (fibrinolisis o angioplastía) y 15\% entre aquellos que no son reperfundidos. Hay una relación directa entre la rapidez en instituir una reperfusión precoz y mejor sobrevida de estos pacientes. Lo mismo es válido para la prevención de la aparición de disfunción ventricular. Tras su egreso hospitalario, el paciente sigue enfrentando una expectativa de vida disminuida en relación con la población normal, observándose entre un 4 y $10 \%$ de mortalidad (de los pacientes que sobrevivieron la hospitalización) durante el primer año, sea a causa de nuevo IAM, insuficiencia cardíaca o arritmias8-10.

En el año 2005 entró en vigencia en nuestro país una ley de Garantías Explícitas en Salud (AUGE), que asegura acceso y financiamiento del tratamiento del IAM en el territorio nacional, prioritariamente mediante trombolisis sistémica. Aunque IOS IAM con SDST tratados con trombolisis aumentaron significativamente en los hospitales públicos, no hay reportes oficiales respecto del impacto de esta ley sobre la morbimortalidad del IAM en el país. En estas pautas se entrega información recogida al respecto por el Grupo de Estudio del IAM (GEMI) de la Sociedad Chilena de Cardiología en algunos hospitales públicos y privados. Las recomendaciones que se entregan en este documento son de carácter científico, e independientes de la normativa AUGE.

Correspondencia: Dr. Eduardo Guarda S.

Depto. Enfermedades Cardiovasculares

Pontificia Universidad Católica de Chile

Marcoleta 367, Santiago

Correo Electrónico: eguarda@med.puc. 


\section{Introducción}

EI IAM con SDST es la manifestación clínica de la oclusión trombótica de una arteria coronaria epicárdica. Con frecuencia la trombosis ocurre en el sitio donde se pierde la integridad una placa aterosclerótica vulnerable ("rotura de placa"), lo cual permite el contacto de la sangre circulante con factores altamente trombogénicos presentes en la placa, tras lo cual se inicia la agregación plaquetaria y se activa la cascada de la coagulación. Más del $90 \%$ de los IAM con SDST ocurren en sitios con estenosis leves a moderadas (menores al $75 \%$ ), las cuales no tienen repercusión hemodinámica y -hasta que se forma el trombo oclusivo- son silentes desde el punto de vista clínico, momento en el cual comienzan a provocar síntomas isquémicos. Como la arteria comprometida tiene escasa o nula circulación colateral, el territorio que irriga se hace rápidamente isquémico, y a los 15-30 min de producida la oclusión comienza la necrosis de la zona correspondiente. La onda de necrosis progresa desde endocardio a epicardio. La reperfusión precoz de la arteria ocluida puede circunscribir la zona de necrosis al subendocardio. Por el contrario, cuando no hay reperfusión se produce una necrosis transmural, la cual puede provocar remodelamiento miocárdico, incluyendo adelgazamiento de la pared y diversos grados de dilatación y disfunción ventricular.

El trombo oclusivo está compuesto por plaquetas y fibrina. De allí que los medicamentos antiplaquetarios, los antitrombínicos y los fibrinolíticos sean esenciales para revertir el proceso. Estos medicamentos pueden lisar el trombo, pero no modifican mayormente la placa aterosclerótica subyacente, razón por la cual el tratamiento de la lesión culpable del IAM suele implicar una angioplastía coronaria (con o sin stent), con la cual se logra reducir significativamente la tasa de reoclusión coronaria.

En estas Pautas se insistirá en que para reducir el daño miocárdico durante el IAM con SDST es fundamental que el diagnóstico del IAM y la terapia de reperfusión se realicen en base a criterios inmediatos, como la anamnesis, el examen físico y el ECG, sin esperar la confirmación enzimática del diagnóstico.

\section{Diagnóstico del IAM}

Un adulto con dolor opresivo retroesternal, con o sin irradiación a los brazos, cuello, espalda o epigastrio, acompañado de sudoración fría y náuseas debe ser transportado a un centro asistencial para confirmar o descartar el diagnóstico de IAM. Para aquellos con angina crónica, un episodio que no ceda 10 minutos post nitroglicerina sublingual también debe motivar el traslado a un centro asistencial. En Chile los reportes del grupo GEMI revelan que en promedio los pacientes consultan alrededor de $4 \mathrm{~h}$ después de comenzado los síntomas, cifra que coincide con otros reportes nacionales. Más preocupante aún, esta demora no se ha reducido en los últimos 7 años11-14. Las mujeres son las que más se demoran en consultar, lo cual también es un problema mundial. Considerando que la mayor mortalidad del IAM ocurre en la primera hora de iniciado los síntomas, y que mayor tiempo con la arteria ocluida significa mayor compromiso de la función ventricular, se requieren mayores esfuerzos para mejorar la educación de la población y de los profesionales de la salud al respecto15,16.

El primer contacto del médico con el paciente con IAM ocurre por lo general en la sala de urgencia o en el domicilio de los pacientes (especialmente en las ciudades que cuentan con unidades móviles). Ante un paciente con dolor torácico sugerente el médico debe realizar una anamnesis dirigida y un ECG dentro de un plazo menor a 10 minutos. Figura 1. Las situaciones clínicas más frecuentes son:

a. Aproximadamente $2 / 3$ de los pacientes presentan un cuadro clínico evidente, caracterizado por: - dolor anginoso característico (opresivo, retrosternal, irradiado a cuello, mandíbula, brazo izquierdo) de más de 30 minutos de duración, acompañado de síntomas neurovegetativos (náuseas, vómitos, sudoración fría, palidez)

- ECG con supradesnivel del segmento ST (IAM con SDST) de más de $1 \mathrm{~mm}$ en al menos dos derivaciones concordantes

- Ante un cuadro clínico y SDST categórico no se requieren más elementos diagnósticos para iniciar la terapia de reperfusión que más convenga al paciente (ver más adelante). 
b. En el tercio restante, el diagnóstico inical no es evidente, ya sea porque los síntomas son atípicos o porque el ECG no es muy claro:

- dolor focalizado en extremidades superiores, dorso, cuello o epigastrio, sin focalización retroesternal.

- ausencia de dolor (frecuente en ancianos y diabéticos). En vez de dolor, el paciente puede presentar disnea o fatiga muscular como síntoma primordial, o insuficiencia cardíaca de instalación brusca.

- al ingreso, el ECG puede ser normal, inespecífico (ej. ondas T «hiperagudas» en el IAM muy inicial), - los cambios isquémicos pueden estar ocultos por alteraciones basales, tales como hipertrofia ventricular izquierda.

- El bloqueo completo de rama izquierda (BCRI) puede enmascarar un IAM con SDST, y por lo tanto se debe intentar precisar si es antiguo o reciente, provocado por el IAM. Si es de reciente aparición, el paciente requerirá reperfusión (ver más adelante).
Estos pacientes sin un diagnóstico claro de IAM pueden estar cursando un IAM sin SDST, cuadro grave, de alta mortalidad, que no será abordado en estas Pautas. Para aclarar el diagnóstico se debe hospitalizar transitoriamente a los pacientes en una Unidad de Dolor Torácico, en la misma sala de emergencia o en una Unidad de Cuidado Intensivo, dependiendo de cada centro. Se deben realizar técnicas diagnósticas adicionales, como la observación evolutiva del ECG, y la determinación seriada de marcadores de necrosis miocárdica, tales como la CK-MB o troponinas, cuya elevación por sobre los rangos normales ocurre en las primeras 6 horas de comenzado el IAM. Las troponinas son el método más sensible y específico para el diagnóstico precoz del IAM.

Con esta rutina se debe descartar el diagnóstico de IAM sin SDST en un plazo no mayor de 12 horas. El tratamiento del Sindrome Coronario Agudo (incluido el IAM sin SDST) puede revisarse en la referencia 17.

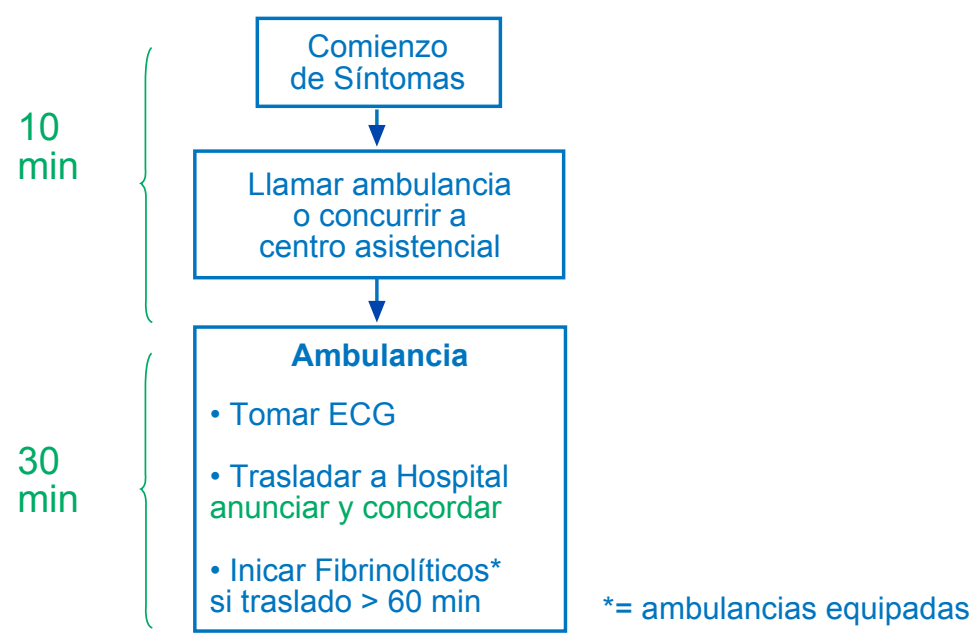

Figura 1: Conducta Prehospitalaria

\section{Criterios de gravedad de un IAM}

Se han identificado las siguientes características como factores de mal pronóstico del IAM:

\section{Criterios clínicos:}

- edad > 65 años

- antecedentes de IAM previo o insuficiencia cardíaca
- diabetes mellitus

- hipotensión al momento del ingreso. Se debe descartar hipotensión por vagotonismo o hipovolemia

- taquicardia > 100 latidos por minuto al ingreso

- presencia de insuficiencia cardíaca aguda. Los pacientes con Killip > I tienen progresivamente peor pronóstico; aquellos en Killip IV (shock cardiogénico) tienen una mortalidad cercana al $70 \%$. 
E. Guarda , J. C. Prieto, P. Sanhueza, C. Dauvergne, R. Asenjo, R. Corbalán.

- Sexo femenino. La mujeres tienen prácticamente el doble de la mortalidad que los hombres

Criterios electrocardiográficos de gravedad de un IAM:

- IAM cara anterior

- Aparición de bloqueo completo de la rama derecha o izquierda en el transcurso de un infarto de pared anterior

- Aparición de bloqueo aurículo-ventricular de 2do o 3er grado, o compromiso del ventrículo derecho en un IAM de cara inferior.

\section{Manejo Pre-hospitalario}

Una vez hecho el diagnóstico de IAM con SDST, el médico debe elegir cuál es la terapia de reperfusión más adecuada para el paciente. Para tomar una buena decisión, el médico debe tener respuesta a las siguientes preguntas:

- ¿Cuánto tiempo ha transcurrido desde el inicio de los síntomas?

- ¿Se puede realizar angioplastía primaria en el centro donde se hizo el diagnóstico?

- ¿Tiene contraindicaciones para fibrinolisis?

- ¿Requiere el paciente traslado en ambulancia a un centro que tenga posibilidades de realizar angioplastía primaria en los siguientes 90 minutos?

Transporte de un paciente con IAM: Si el diagnóstico de un IAM con SDST se ha hecho en un recinto extrahospitalario (ambulancia, consultorio médico), hay que trasladar al paciente para una pronta terapia de reperfusión (trombolisis o angioplastía primaria). De ser posible, el paciente debe ser trasladado a un centro donde se pueda realizar angioplastía primaria antes de 90 minutos. Ver Figura 2.

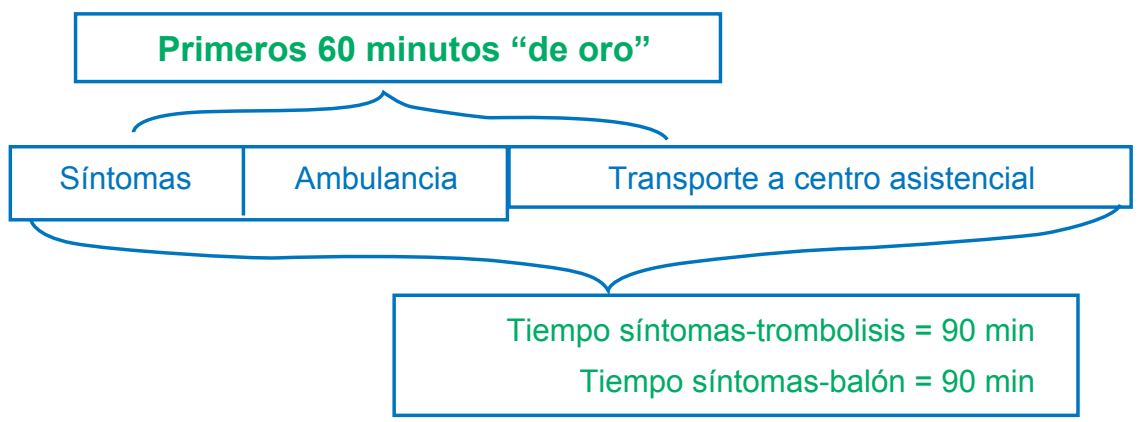

Tiempo de isquemia total $=90 \mathrm{~min}$

Figura 2: Tiempos Ideales Prohospital

Esta conducta otorga mejores resultados que la trombolisis (ver más adelante). En cambio, si se presume que el traslado implicará una demora mayor que la señalada, o si no hay disponibilidad inmediata de angioplastía, debe iniciarse la trombolisis antes de $30 \mathrm{~min}$ de confirmado el diagnóstico, en el lugar donde se disponga de las condiciones mínimas para realizar la infusión 1-5.

Como primera medida ante la sospecha de un
IAM, dar una tableta de $500 \mathrm{mg}$ de aspirina a masticar antes del traslado.

Fibrinolisis pre-hospitalaria: Un metaanálisis reciente 18 demostró que el inicio de trombolisis en ambulancias equipadas antes de llegar al hospital puede reducir en un $17 \%$ la mortalidad, comparado con fibrinolisis en el hospital. En esos ensayos se insistió en que el mejor efecto se consigue cuando la 
trombolisis se inicia antes de 2 horas de comenzados los síntomas, por lo cual sigue siendo importante que el paciente o sus familares llamen precozmente al servicio de ambulancias ${ }^{19-22}$. La implementación de ambulancias equipadas y con personal entrenado para efectuar la trombolisis en el lugar del diagnóstico, antes de llegar al hospital base, se justificaría en lugares del país donde el transporte a un centro asistencial fuera mayor de $60 \mathrm{~min}$. Este es un tema de gran importancia social, puesto que los pacientes que viven en sitios muy alejados de los centros urbanos hasta hoy enfrentan enormes dificultades para ser atendidos en los plazos requeridos. Por otro lado, es necesario entender que implementar medidas de esta envergadura requiere un alto grado de entrenamiento y capacitación del personal de ambulancias.

\section{Tratamiento del IAM con SDST durante las primeras 24 horas}

Las medidas generales deben comenzar en el lugar donde se hace el diagnóstico de IAM, vale decir, en el domicilio del paciente (ambulancias equipadas) o en la sala de emergencia.

Específicamente, no debe esperarse tener al paciente en la Unidad Coronaria o en Intensivo para iniciar las medidadas generales o la trombolisis. Si se optó por angioplastía, el paciente debería ser llevado directamente desde la sala de emergencia a una sala de hemodinamia.

a) Medidas generales ante un paciente con IAM con SDST:

1. Reposo absoluto en cama

2. Aspirina ( AAS ): Dar una tableta de $500 \mathrm{mg}$ a masticar, si aún no la ha recibido. No usar las tabletas con recubierta entérica debido a que su absorción es más lenta.

3. Instalar dos vías venosas periféricas en distintos antebrazos. No usar medicamentos vía intramuscular en el curso del IAM.

4. Régimen 0 durante las primeras 12 horas y líquido durante las 12 horas siguientes.

5. Monitorización y hospitalización en Unidad que cuente con desfibrilador y carro de paro cardíaco

6. Oxígeno por naricera 2 a $4 \mathrm{lt} / \mathrm{min}$, idealmente en un flujo suficiente como para mantener una saturación $>92 \%$, medida con oximetría de pulso.

7. Iniciar Terapia de reperfusión (ver detalles más adelante)

8. Aporte de volumen IV, ya que la mayoría de los pacientes con IAM presenta hipovolemia, la que puede ser importante especialmente en aquellos pacientes que han presentado sudoración y vómitos profusos y que ingresan con presión arterial sistólica $<100 \mathrm{mmHg}$, en ausencia de signos congestivos.

9. Alivio inicial del dolor: la morfina IV 2 a $4 \mathrm{mg}$ en bolo constituye un analgésico eficaz y seguro, salvo en pacientes hipovolémicos o con IAM inferior con compromiso del ventrículo derecho (ver más adelante). Puede repetirse la dosis cada $15 \mathrm{~min}$. La morfina puede inducir vómitos e hipotensión, por lo cual su uso debe ser ciudadoso. No administrar antiinflamatorios no específicos (AINEs) durante la hospitalización, en especial los inhibidores de la COX-2. En el caso de que los pacientes consuman habitualmente AINEs (exceptuando aspirina), al momento del IAM deberán suspenderse, debido a que aumentan el riesgo de hipertensión arterial, muerte, reinfarto, falla cardíaca y rotura de miocardio 23,24 .

10.Nitroglicerina: constituye un efectivo antianginoso en muchos pacientes. Por su capacidad de reducir la precarga ventricular y de vasodilatar el árbol coronario, la nitroglicerina disminuye el consumo de oxígeno miocárdico. Está especialmente indicada en pacientes con evidencias de disfunción ventricular y congestión pulmonar en el curso del IAM, en pacientes hipertensos, o con angor persistente. La nitroglicerina está contraindicada en pacientes con presión arterial sistólica menor de $90 \mathrm{mmHg}$ (especialmente en pacientes con frecuencia cardíaca bajo 60 o sobre 100 latidos / minuto) y en sujetos con infarto ventricular derecho, casos en los que su uso puede ser deletéreo. Si bien puede utilizarse 
inicialmente una dosis sublingual en el box de urgencia, en el paciente hospitalizado debe administrarse por vía intravenosa con bomba de infusión contínua en dosis inicial de 5 a $10 \mu \mathrm{g} / \mathrm{min}$ y con incrementos de $10 \mu \mathrm{g} /$ minuto cada 15 minutos hasta obtener una reducción de la presión arterial media de aproximadamente $10 \%$ o más, en el paciente que persiste hipertenso. La vía transdérmica y las formulaciones orales de acción prolongada no se recomiendan en esta fase, por el riesgo de inducir hipotensión.

11. Betabloqueadores: Por el dolor y la ansiedad, es frecuente que el paciente con IAM tenga una importante descarga adrenérgica, la cual puede gatillar arritmias y aumentar el consumo de oxígeno. A pesar de esto, no hay evidencia que respalde el uso de betabloqueadores IV en las primeras horas; de hecho, su uso en pacientes con hemodinamia límite puede desencadenar hipotensión o falla ventricular izquierda. Por lo tanto, el uso de betabloqueadores IV (propranolol, metoprolol) debe restringirse a pacientes con taquicardia sinusal e hipertensión arterial que no tengan evidencias de insuficiencia cardíaca aguda25,26. Aún así, se debe estar muy atento para pesquisar inmediatamente la aparición de bloqueo AV o congestión pulmonar, especialmente ante infartos extensos de cara anterior. En caso de duda, la función ventricular global puede ser evaluada mediante Eco 2D. En cambio, la utilidad de los betabloqueadores vía oral a partir del segundo día en pacientes estables ha sido claramente demostrada. Ver sección "control de factores de riesgo". Si el paciente recibe betabloqueadores en forma crónica (previo al IAM), se debe intentar mantener la indicación, disminuyendo la dosis a la mitad si la situación clínica lo permite. Los betabloqueadores están contraindicados ante evidencia clínica de compromiso severo de la función ventricular (ritmo de galope, congestión pulmonar), bloqueo aurículo-ventricular de $2^{\circ}$ y $3^{\circ}$ grado, o bloqueo AV simple con $P R>0.24$ segs e hiperreactividad bronquial.

12. Clopidogrel: Los estudios COMMIT-CCS-2 27 y Clarity TIMI 2828 demostraron la utilidad del antiplaquetario clopidogrel en el IAM con SDST, ya que reduce la mortalidad global, el reinfarto y aumenta la tasa de arterias permeables, sin aumentar las hemorragias.

En el caso de trombolisis, se debe usar una dosis de carga de $300 \mathrm{mg}$, seguido de $75 \mathrm{mg}$ día. En mayores de 75 años no se recomienda administrar dosis de carga 28 . En el caso de angioplastía primaria, la dosis de carga es de $600 \mathrm{mg}$, independiente de la edad1,2,4,5,29.

No existe evidencia clínica sobre por cuánto tiempo usar clopidogrel post trombolisis. Las guías europeas y americanas sugieren mantener la terapia hasta por 1 año.

13. Inhibidores de la enzima convertidora de angiotensina (IECA) e Inhibidores del receptor de Angiotensina II (ARAII): la administración de estos medicamentos previene la dilatación ventricular y reduce la mortalidad en pacientes con infartos extensos 30 . Sin embargo, su empleo durante las primeras 24 horas puede complicar el manejo del paciente, al sumarse su acción hipotensora a la de otros fármacos como la nitroglicerina los betabloqueadores, morfina y la estreptokinasa (SK). Se recomienda iniciar estos medicamentos una vez que el paciente se encuentra estabilizado, con presión arterial sistólica sobre $100 \mathrm{mmHg}$, con fármacos de acción corta y en dosis crecientes (ejemplo captopril $3.125 \circ 6.25 \mathrm{mg}$ cada $6-8 \mathrm{hrs}$ ), subiendo la dosis de acuerdo con la tolerancia clínica.

En caso de intolerancia a los IECA (alergia, tos irritativa), pueden utilizarse los ARA II, iniciándolos a dosis bajas.

14. Diuréticos y Antagonistas del calcio: Debe evitarse el uso de diuréticos en las primeras horas de un IAM, ya que la mayoría de los pacientes presentan hipovolemia. Un error frecuente es la administración de diuréticos a sujetos con hipertensión arterial durante la crisis de dolor, la cual puede ser secundaria a vasoconstricción. En estos casos la administración de diuréticos puede provocar caída brusca de la presión arterial sistólica y agravar 
el curso del IAM. Lo que se recomienda en esos casos es tratar el dolor con morfina y TNT IV. Sólo debiera usarse furosemida IV en dosis bajas en casos de congestión pulmonar sintomática.

Los antagonistas del calcio no han demostrado ser de beneficio en pacientes con IAM con $\mathrm{SDST}^{31}$. Son de riesgo en pacientes con falla ventricular izquierda. Su uso está restringido a pacientes con isquemia persistente a pesar de terapia médica óptima y que no pueden usar betabloqueadores por broncoespasmo. En este caso, se puede utilizar diltiazem y verapamilo.

15.Estatinas: Su uso en prevención primaria y secundaria está claramente demostrado. Respecto de su uso en el IAM, recientemente se ha señalado que las estatinas que más disminuyen el colesterol LDL reducen leve, pero significativamente, el reinfarto y los accidentes vasculares oclusivos, sin afectar la mortalidad cardíaca (32). Hay consenso en que a todos los pacientes se les debiera medir el perfil lipídico durante las primeras $24 \mathrm{~h}$ del IAM, y que debieran recibir estatinas en el momento del alta. Las recomendaciones internacionales señalan que el colesterol LDL debe ser $<70-80 \mathrm{mg} / \mathrm{dl}$ en esta población de alto riesgo3,5. Ver más detalles en "Tratamiento de los factores de riesgo".

\section{Reperfusión Coronaria}

En el paciente que consulta en el transcurso de las primeras 12 horas, el objetivo terapéutico primario consiste en reestablecer el flujo coronario del vaso ocluido en la forma más rápida posible. Durante la última década -y luego de muchas campañas educativas- se ha logrado reducir en 30 minutos el tiempo para iniciar la trombolisis o realizar la angioplastía primaria, lo que ha significado una reducción de la mortalidad de un 1 y $5 \%$, respectivamente.

En nuestro país, de acuerdo al GEMI, hasta el 40\% de los pacientes no recibe terapia de reperfusión. La principal causa es el ingreso tardío (> $12 \mathrm{~h}$ ) del paciente, pero en otros casos se debe a contraindicación de uso de trombolíticos, o a hipotensión marcada secundaria a la infusión de SK, sin que el paciente sea trasladado a un centro capacitado para realizar angioplastía primaria. Se comentará en esta sección que los pacientes que no reciben trombolíticos tienen peor pronóstico, y que se debe contar con mecanismos que permitan trasladarlos a un centro que cuente con hemodinamia.

Las 2 terapias de reperfusión más utilizadas son la trombolisis sistémica y la angioplastía coronaria.

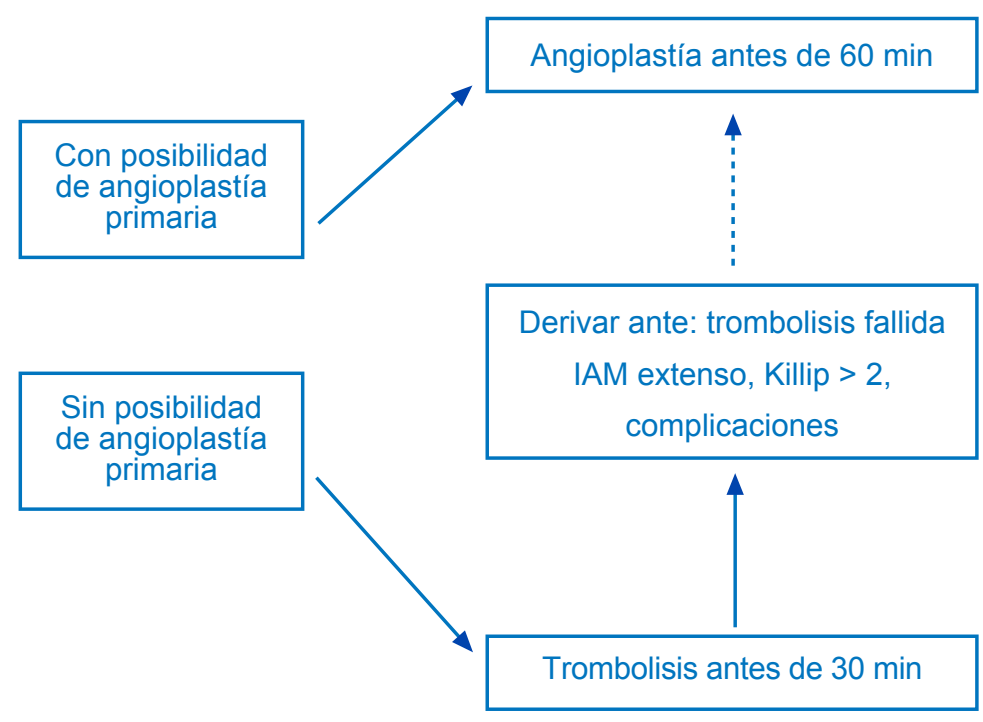

Figura 3: Acciones en el Hospital que recibe al paciente con IAM con SDST 


\section{Consideraciones respecto de la mejor terapia de reperfusión}

Si el paciente ingresa con un IAM con SDST $<2$ horas de evolución, las diferencias en cuanto a mortalidad entre trombolisis y angioplastía son mínimas. Si el paciente llega con más de 3 horas de evolución, la angioplastía primaria tiene clara ventaja respecto de la trombolisis 33 . Como en Chile el diagnóstico se hace alrededor de las 4 horas de iniciado los síntomas, la mayoría de los pacientes debieran ser tratados con angioplastía primaria12, 34-38. Figura 3.

Otros casos en donde la angioplastía es claramente favorable por sobre la trombolisis son los IAM graves (IAM extenso de cara anterior, shock cardiogénico, Killip e III), IAM en personas > 75 años, y obviamente cuando la trombolisis tiene una contraindicación formal Ocasionalmente no hay claridad diagnóstica absoluta. Por ejemplo, un paciente con dolor sugerente y SDST mínimo. En esos caso dudosos, es preferible una coronariografía diagnóstica (y eventual angioplastía, si se confirma la oclusión trombótica) que una trombolisis en un paciente que no la requiere o está contraindicada (pericarditis, disección aórtica).

El criterio más pragmático para elegir la terapia de reperfusión es la posibilidad real o no de realizar angioplastía primaria en el centro donde se hizo el diagnóstico. En un centro que no cuente con angioplastía primaria se debe inicar trombolisis a menos que el paciente pueda ser trasladado a un centro que inicie la angioplastía antes de 90 min 39-41. Figura 4.

\begin{tabular}{|c|c|c|c|}
\hline & & $\begin{array}{l}\text { Paso 1: Definir tiempo de evolución y riesgo del IAM } \\
\text { - Cuánto tiempo ha transcurrido desde el inicio de los síntomas? } \\
\text { - Cuán grave es el IAM? } \\
\text { - Riesgo de trombolisis? } \\
\text { - Posibilidades de traslado del paciente en plazo aceptable? }\end{array}$ & \\
\hline & & \multicolumn{2}{|c|}{ Paso 2: Definir el tipo de Revascularización: } \\
\hline & $\begin{array}{l}\text { Fibrinolisis como primera elección: } \\
\text { Menos de } 2 \text { horas de iniciado los } \\
\text { síntomas e imposibilidad de angioplastía } \\
\text { en menos de } 60 \text { min en el lugar de } \\
\text { ingreso, o de } 90 \mathrm{~min} \text {, si hay necesidad } \\
\text { de traslado. }\end{array}$ & \multicolumn{2}{|c|}{$\begin{array}{l}\text { Angioplastía como primera elección } \\
\text { - En todo hospital que tenga personal } \\
\text { entrenado y con posibilidad de realizar la } \\
\text { angioplastía antes de } 60 \text { min ("puerta-balón" } \\
<60 \text { min) } \\
\text { - Angioplastía antes de } 90 \text { min si el paciente } \\
\text { puede ser trasladado desde otro centro } \\
\text { (“contacto médico-balón" < } 90 \text { min), } \\
\text { especialmente ante infartos de alto riesgo: } \\
\text { - Shock cardiogénico } \\
\text { - Killip } \geq 3 \\
\text { - Presentación tardía (síntomas }>2 \text { h) } \\
\text { - Con contraindicaciones a la fibrinolísis (ver figura 5) } \\
\text { - Frente a duda diagnóstico del IAM }\end{array}$} \\
\hline
\end{tabular}

Figura 4: Estrategias de Reperfusión en pacientes con IAM con SDST

En caso de indicar el traslado, el médico a cargo debe tener en cuenta variables médicas y no médicas. Entre las primeras, el estado del paciente, la calidad del equipamiento de la ambulancia donde se hará el traslado y, muy importantemente, deberá contactar al hemodinamista que realizará la angioplastía, para asegurarse de que el enfermo no tendrá que esperar para entrar a la sala de hemodinamia. Entre las segundas, deberían existir acuerdos previos entre los centros que derivan y reciben al paciente, para no tener trabas administrativas al ingreso. Un detalle importante en las grandes ciudades es el estado del tránsito a la hora del traslado, puesto que las congestiones vehiculares pueden retrasar el inicio de la angioplastía.

En nuestro país se analizaron datos de 3.255 pacientes que ingresaron con IAM con SDST $<12 \mathrm{~h}$ a la red GEMI entre los años 2001-200514. La mortalidad global fue de $9,9 \%(7,5 \%$ en hombres y $16,7 \%$ en mujeres; $p<0.0001)$. Los pacientes 
tratados con tromboliticos $(n=1.652)$ tuvieron una mortalidad de $10,2 \%$ ( $7,6 \%$ en hombres y $18,7 \%$ en mujeres; $p<0,0001)$; en los sometidos a angioplastía primaria $(n=468)$ fue de $4,7 \%(2,5 \%$ en hombres $y$ $13 \%$ en mujeres; $p<0,0001$ ), y en los que no recibieron terapia de reperfusión $(n=1.135)$ fue de $11,6 \%$ $(9,8 \%$ hombres y $15,4 \%$ en mujeres; $p=0,006)$. Por lo tanto, parece evidente que en nuestro país la angioplastía primaria debe ser el procedimiento de revascularización de elección en pacientes con IAM, tanto en hombres como en mujeres.

Cualquiera sea la decisión, esta deberá ser tomada con rapidez, para lograr el objetivo de salvar la mayor cantidad posible de músculo cardíaco. Los centros deben realizar auditorías internas al menos 2 veces al año para asegurarse de que están cumpliendo con los plazos recomendados: fibrinolítico antes de $30 \mathrm{~min}$ o angioplastía antes de 60 minutos de ingresado al hospital, o antes de $90 \mathrm{~min}$, en el caso de traslados desde otro centro.

Lamentablemente, como se señaló previamente, en nuestro país el porcentaje de pacientes con IAM con SDST en quienes no se realiza terapia de reperfusión (trombolisis o angioplastía) alcanza al $40 \%$. La principal causa de la no utilización de un procedimiento de reperfusión en nuestro país es el diagnóstico tardío. Esto, a su vez, tiene varias explicaciones, todas ellas remediables: demora en concurrir al servicio de urgencia, lentitud en la realización de un ECG que corrobore el diagnóstico, y finalmente el no traslado del paciente a un centro que cuente al menos con posibilidades de realizar trombolisis.

\section{b) Angioplastia Primaria en el IAM con SDST}

La angioplastía primaria o directa es la terapia de reperfusión de elección en el IAM con SDST. Tanto la literatura mundial como las cifras nacionales concluyen que la mortalidad del IAM tratado con angioplastía primaria es significativamente menor que con trombolisis sistémica. La angioplastía también tiene ventajas en cuando a una menor incidencia de reinfarto y de accidente vascular hemorrágico. La menor incidencia de complicaciones intrahospitalarias y de readmisiones se debería a una menor incidencia de isquemia residual y de reoclusión de la arteria culpable 33 .

Cifras internacionales señalan que la angioplastía primaria permite restablecer flujo coronario adecuado (TIMI III) en prácticamente el $90 \%$ de los pacientes tratados. En cambio, los trombolíticos fibrino específicos, como tPA y tenecteplase (TNK) alcanzan flujo TIMI III en el $65 \%$ de los casos. Además, la angioplastía deja a la arteria sin estenosis residual, a diferencia de los trombolíticos, que no modifican la estructura de la placa ateromatosa 42,43 . Cifras nacionales señalan que con angioplastía primaria se alcanza Flujo TIMI 3 en el 87\%, y TIMI 2 en el 10\%34,35,37. En cambio, con trombolisis con estreptoquinasa (SK) en nuestro país se obtuvo flujo TIMI III en el $42 \%$, cifra por lo demás, semejante a la experiencia internacional $(45 \%)$, observándose además una estenosis residual $>70 \%$ en el $49 \%$ de los pacientes 43 .

Utilizando los registros nacionales de angioplastía coronaria (RENAC) y de trombolisis (GEMI), se compararó la mortalidad de los pacientes con IAM con SDST que recibieron trombolisis (857 pts) versus aquellos que fueron tratados con angioplastía primaria, en un seguimiento hasta 24 meses 35 .

Fueron incluidos todos los pacientes de ambos registros tratados durante los años 2003 y 2004 . La mortalidad de la angioplastía a 30 días fue de $8.1 \%$ versus $11.1 \%$ en los tratados con fibrinolíticos $(p<0.01)$, y a 24 meses de 11.7 vs $21 \%$, respectivamente $(p<0.001)$. Por lo anteriormente expuesto, parece claro que en nuestro país la angioplastía primaria debería ser la terapia de reperfusión de elección en el IAM con SDST.

Consideraciones logísticas, sin embargo, determinan que la angioplastía primaria no sea la terapia más utilizada aún en los países desarrollados. En USA, por ejemplo, sólo el $25 \%$ de los hospitales tienen laboratorio de hemodinamia, y se estima que las terapias de reperfusión del IAM con SDST corresponden $10 \%$ angioplastía y $60 \%$ a trombolisis, mientras que aproximadamente $1 / 3$ de los pacientes no recibe terapia de reperfusión 
E. Guarda , J. C. Prieto, P. Sanhueza, C. Dauvergne, R. Asenjo, R. Corbalán.

alguna44. Además, sólo alrededor del $5 \%$ de los pacientes son tratados dentro de los primeros $90 \mathrm{~min}$ y $16 \%$ en los primeros $120 \min 45$. En Chile se dispone de cineangiógrafos sólo en Iquique, Antofagasta, Valparaíso, Viña del Mar, Santiago, Rancagua, Talca, Concepción, Temuco, Puerto Montt y Punta Arenas. Se debería contar con al menos 1 cineangiógrafo en cada Región para mejorar el acceso a esta tecnología a lo largo del país. Con programas regionales específicos que busquen optimizar el tratamiento del IAM con SDST es posible reducir significativamente la mortalidad del IAM46.

Además de su alto costo operacional, para que sea efectiva, la angioplastía debe ser realizada por operadores con amplia experiencia antes de 90 minutos desde el ingreso del paciente. Se ha definido que para que un operador sea "experto" debe realizar al menos 75 angioplastías al año. El personal del laboratorio también ha de tener amplia experiencia, de modo que el centro realice al menos 200 angioplastías por año.

Una vez decidida la angioplastía primaria, los pacientes deben recibir aspirina no recubierta $500 \mathrm{mg}$, clopidogrel $600 \mathrm{mg}$ y heparina no fraccionada $(100 \mathrm{Ul} / \mathrm{kg}$ bolo IV; $60 \mathrm{UI} / \mathrm{kg}$ si se administra con abciximab, tirofiban o eptifibatide). En más del $90 \%$ de los casos se implanta un stent coronario. El uso de abciximab (antagonista IIb/llla) durante la angioplastía ha demostrado utilidad cuando existe una gran carga trombótica en el vaso culpable, con reducción de la mortalidad según una reciente revisión sistemática de la literatura47. En cambio, su uso antes de conocer la anatomía coronaria no es de utilidad48-49. Asimismo, el estudio ASSENT-4 PCI 50 demostró que la angioplastía facilitada (uso rutinario de trombolíticos previo a la angioplastía) tenía mayor mortalidad que realizar la angioplastía sin uso previo de trombolíticos.

En pacientes que han recibido trombolíticos, la angioplastía debe plantearse también frente a casos de:

- Fracaso de la trombolisis (ausencia de signos de reperfusión 90 min después de finalizada la administración de SK) en pacientes con infartos extensos, con síntomas persistentes y que están aún dentro del plazo de 12 horas (angioplastía de rescate). La evidencia muestra que la angioplastía de rescate reduce significativamente la tasa de eventos tales como mortalidad, reinfarto, insuficiencia cardíaca, comparada con una nueva fibrinolisis o tratamiento no intervencional 51 .

- Reinfarto en pacientes previamente sometidos a trombolisis

- Pacientes con deterioro hemodinámico post trombolisis, especialmente si se desarrolla un shock cardiogénico: la angioplastía está indicada en pacientes < 75 años con infartos con SDST en Killip IV con menos de 36 horas de evolución y menos de 18 horas de shock52. El uso de balón de contrapulsación intraaórtico es fundamental en estos casos. Con frecuencia, la angioplastía del vaso culpable debe ser acompañada de angioplastía de otros vasos, para mejorar la contractilidad de esos territorios en pacientes con enfermedad de múltiples vasos. En otras ocasiones, la anatomía coronaria hace necesaria una cirugía de revascularización.

\section{C) Fibrinolisis}

Si no es posible realizar angioplastía antes de 90 min después de hecho el diagnóstico de IAM con SDST, debe realizarse una trombolisis sistémica. Tal como para el caso de la angioplastía primaria, la decisión de administrar trombolíticos debe basarse en criterios inmediatos como la anamnesis, el examen físico y el ECG. Cuando los tres son concordantes, se debe iniciar de inmediato la trombolisis, a menos que exista alguna contraindicacion. No se debe esperar a la confirmación enzimática del diagnóstico ni los pacientes deben ser trasladados a una unidad especializada: en lo posible la administración del trombolitico debe realizarse en el servicio de urgencia que recibe al paciente. Debe verificarse que el paciente no tenga contraindicaciones al uso de trombolíticos $53-55$ (figura 5). 


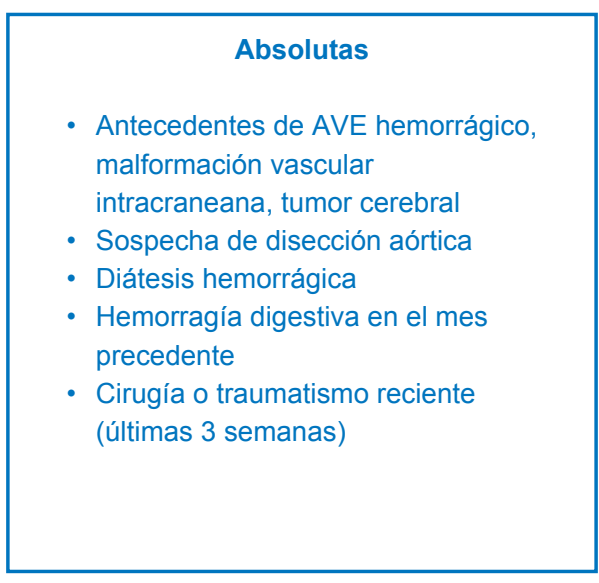

Figura 5: Contraindicaciones a la Trombolisis

En pacientes con clínica sugerente de IAM, pero cuyo ECG demuestra bloqueo completo de rama izquierda (no presente en ECG previos), los trombolíticos no están contraindicados. Estos paciente pueden ser un subgrupo particularmente

\begin{tabular}{|l|}
\hline \multicolumn{1}{|c|}{ Relativas } \\
- Hipertensión arterial al ingreso > 180 / \\
$110 \mathrm{~mm} \mathrm{Hg}$ \\
- Maniobras de resucitación cardiopulmonar \\
$>10$ min \\
- Embarazo, y post parto hasta 3 meses \\
- Uso SK últimos 5 días (riesgo anafilaxia) \\
- Punción de vaso en sitio no comprensible \\
(vena subclavia) \\
- AVE isquémico en los últimos 6 meses \\
- Tratamiento anticoagulante: mientras mayor \\
el INR, mayor el riesgo de hemorragia.
\end{tabular}

beneficiado.

En cambio, en los IAM sin SDST, la trombolisis no está indicada; no se ha demostrado beneficio en este subgrupo de pacientes.

Esquemas de uso de Fibrinolíticos (TABLA 1)

Tabla 1: Dosis de Fibrinolíticos en el IAM con SDST

\begin{tabular}{|l|l|}
\hline Estreptokinasa & 1.5 millones U en $45 \mathrm{~min}$ \\
\hline Alteplase (t-PA) & $\begin{array}{l}15 \mathrm{mg} \text { bolo IV. seguido por } 0.75 \mathrm{mg} / \mathrm{kg} \text { IV en } 30 \mathrm{~min} \text { y luego } 0.5 \mathrm{mg} / \mathrm{kg} \\
\text { IV en } 60 \mathrm{~min} . \text { NO exceder } 100 \mathrm{mg} / \mathrm{total}\end{array}$ \\
\hline Reteplase (r-PA) & $10 \mathrm{U}+10 \mathrm{U} \mathrm{IV}$. separados por $30 \mathrm{~min}$ \\
\hline Tenecteplase (TNK) & $\begin{array}{l}\text { Bolo IV único en } 10 \mathrm{seg}: 30 \mathrm{mg} \text { en }<60 \mathrm{~kg} ; 35 \mathrm{mg} \text { entre } 60-69 \mathrm{~kg}: 40 \mathrm{mg} \\
\text { ente } 70-79 \mathrm{~kg}, 45 \mathrm{mg} \text { entre } 80-90 \mathrm{~kg} \text { y } 50 \mathrm{mg} \text { para personas }>90 \mathrm{~kg}\end{array}$ \\
\hline
\end{tabular}

Estreptokinasa: A pesar de no ser el mejor de su clase, es el fibrinolítico más ampliamente utilizado en el país:

- Diluir 1.500.000 U.I. de SK en solución salina al 9/oo ("suero fisiológico", $250 \mathrm{ml}$ ).

- Infundir esta solución por vía intravenosa en aproximadamente 45 minutos. En ciertos centros la droga se infunde a mayor velocidad (en $30 \mathrm{~min}$ ), pero aumenta la incidencia de hipotensión.

Precauciones al usar SK :

* La hipotensión arterial (producto de una caída en la resistencia vascular sistémica) se presenta en aproximadamente un $20 \%$ (reporte de grupo GEMI), por lo que es conveniente controlar la presión arterial del paciente cada 5 minutos a lo menos durante los primeros 15 minutos de infusión de SK. Por lo general, la hipotensión responde rápidamente a:

- suspensión transitoria de la infusión de SK (y Nitroglicerina)

- elevación de extremidades inferiores

- administración rápida de suero fisiológico y Atropina.

* Reacciones alérgicas cutáneas, las cuales son infrecuentes y por lo general, no graves. Ante la sospecha de una reacción alérgica debe suspenderse la SK y administrarse Clorprofenpiridamina 
E. Guarda , J. C. Prieto, P. Sanhueza, C. Dauvergne, R. Asenjo, R. Corbalán.

$10 \mathrm{mg}$ IV y eventualmente Hidrocortisona $100 \mathrm{mg}$ IV.

Activador tisular del plasminógeno (tPA, alteplase): Bolo intravenoso de $15 \mathrm{mg}$ seguido por una infusión de $0.75 \mathrm{mg} / \mathrm{Kg}$ ( $\sin$ exceder $50 \mathrm{mg}$ ) en $30 \mathrm{~min} y$ luego $0.5 \mathrm{mg} / \mathrm{Kg}$ (sin exceder $30 \mathrm{mg}$ ) en $60 \mathrm{~min}$, hasta una dosis máxima de $100 \mathrm{mg}$ en $90 \mathrm{~min}$.

Tenecteplase (TNK): bolo de $30 \mathrm{mg}$ en $10-15$ seg en $<60 \mathrm{~kg}$; $35 \mathrm{mg}$ entre $60-69 \mathrm{~kg} ; 40 \mathrm{mg}$ entre $70-79 \mathrm{~kg}$, $45 \mathrm{mg}$ entre $80-90 \mathrm{~kg}$ y $50 \mathrm{mg}$ para personas $>90 \mathrm{~kg}$. Sus principales ventajas respecto de SK es que basta un solo bolo IV, administrado en segundos (versus 45 min de la SK), no induce hipotensión ni reacciones alérgicas y las tasas de reperfusión son mayores que con la SK56, 57.

Tanto el tPA como en TNK deben usarse en forma concomitante con heparina fraccionada $60 \mathrm{U} / \mathrm{kg}$ hasta un máximo de $4.000 \mathrm{U}$, seguida de infusión inicial de hasta $12 \mathrm{U} / \mathrm{kg} / \mathrm{h}$, máximo $1000 \mathrm{U} / \mathrm{h}$, regulando según TTPA o ACT.

Por las razones enunciadas, por su simplicidad de administración y su mayor eficacia, proponemos que en nuestro país tenecteplase reemplace a la SK como el trombolítico de elección. Con los fibrinolítico específicos (t-PA, TNK) se consigue flujo TIMI III de la arteria culpable en el $60 \%$ - $65 \%$ de los casos, bastante mejor que con la SK (40-45\%). 44

Complicaciones con el uso de fibrinolíticos:

- La administración de fibrinolíticos se asocia con un riesgo de hemorragias mayores (que requieren de transfusión o cirugía) en 1 - $6 \%$ de los pacientes. La mayoría ocurre en sitios de punción. Por lo mismo, debe evitarse la punción de arterias o venas centrales. En caso de requerirse la instalación de una sonda estimuladora de marcapasos u otro dispositivo por vía venosa central, se recomienda efectuarla por vía femoral (sitio compresible) y bajo visión radioscópica.

- La complicación más temida es el accidente vascular encefálico hemorrágico, que se presenta en aproximadamente $0.4-1 \%$ de los pacientes tratados con SK. La edad avanzada
(>75 años), peso bajo, sexo femenino e hipertensión arterial se asocian con un mayor riesgo de hemorragia. Una de las pocas ventajas que tendría la SK respecto de los fibrinolíticos específicos es que los pacientes mayores de 75 años podrían tener menor riesgo de hemorragia cerebral con este medicamento, sobre todo si se la utiliza a mitad de la dosis habitual.

- El uso concomitante de heparina con SK no ha probado ser beneficiosa en reducir la mortalidad. Si se decide usar heparina, ésta puede iniciarse aproximadamente a las 6 horas post SK, manteniendo su infusión por 48 horas con niveles de ACT de $250 \mathrm{seg}$ o TTPA de $70-80 \mathrm{seg}$.

El empleo de la asociación de trombolíticos específicos en dosis reducidas con antagonistas del receptor IIb/IIla plaquetario no ha demostrado ser de utilidad clínica, por lo cual no deben usarse.

Los signos de reperfusión más útiles para la interpretación del resultado de la trombolisis son:

* Disminución significativa (50\%) del dolor durante los primeros $90 \mathrm{~min}$ de iniciada la infusión del trombolítico. Para poder comparar los efectos de la terapia, debe hacerse una graduación del dolor (escala de 1 a 10) al ingreso del paciente, inmediatamente antes del inicio y cada 5 min después de comenzada la infusión.

* Caída del supradesnivel ST en más de un $50 \%$ dentro de los primeros $90 \mathrm{~min}$ de administrado el trombolítico.

* Inversión precoz (dentro de las primeras 24 horas) de la onda $T$ en las derivaciones electrocardiográficas en que se manifiesta el IAM.

* Peak enzimático precoz, antes de las 12 horas de iniciada la trombolisis.

La utilidad clínica de estos signos aumenta cuando coexisten 3 criterios en forma simultánea. Las «arritmias de reperfusión» no son específicas ni sensibles como parámetro de resultado de la trombolisis sistémica. La presencia de estos índices 
no invasivos de reperfusión se asocian a un mejor pronóstico intrahospitalario y alejado.

\section{Drogas Asociadas al uso de Fibrinolíticos:}

Aspirina (AAS): todos los fibrinolíticos deben administrarse conjuntamente con Aspirina (250 a $500 \mathrm{mg}$ de cualquier formulación no recubierta, a masticar). La asociación de SK y Aspirina prácticamente duplica la efectividad de la SK sola, en términos de reducir la mortalidad. La Aspirina es efectiva también por si sola (independiente de la administración de SK ) y su uso está indicado en todo paciente con IAM salvo alergia grave o úlcera péptica sangrante.

Nitroglicerina y Betabloqueadores: Deben emplearse según las pautas y con las precauciones ya señaladas.

Antitrombóticos: La administración simultánea de trombolíticos y heparina no fraccionada por vía intravenosa aumenta el porcentaje de arterias permeables en los pacientes que reciben fibrinolíticos específicos (tPA, reteplase o tenecteplase), pero no en los que reciben SK. En este último caso, la asociación con heparina no modifica significativamente la mortalidad, pero disminuye el riesgo de reinfarto, a costa de aumentar el riesgo de hemorragias mayores. El riesgo de accidente vascular encefálico hemorrágico (aproximadamente $0.45 \%$ con SK- Aspirina) se eleva a $0.55 \%$ al a gregar heparina IV. El uso de heparina no fraccionada con posterioridad a la SK está indicado en pacientes con alto riesgo de embolía sistémica, tales como IAM extenso de cara anterior, fibrilación auricular o visualización de trombos intraventriculares por ECO-2D.

Con los fibrinolíticos específicos se recomienda usar heparina hasta $48 \mathrm{~h}$ post IAM. En el estudio ASSENT-3 el tratamiento con tenecteplase más enoxiparina disminuyó el riesgo de reinfarto durante el primer mes, comparado con heparina no fraccionada57. Más recientemente se demostró que fondaparinux, un agente anti $\mathrm{Xa}$, disminuyó muerte y reinfarto (versus heparina no fraccionada) en pacientes post trombolisis (OASIS-6, ref 58). Tabla 2.

Tabla 2: Dosis de Antitrombínicos en el IAM con SDST

\begin{tabular}{|c|c|}
\hline \multicolumn{2}{|l|}{ En Angioplastía Primaria } \\
\hline - Heparina No fraccionada & $\begin{array}{l}100 \mathrm{u} / \mathrm{kg} \text { bolo IV; } 60 \mathrm{U} / \mathrm{kg} \text { si se administra con abciximab, } \\
\text { tirofiban o eptifibatibe }\end{array}$ \\
\hline - Bivalirubina & $\begin{array}{l}\text { Bolo IV } 0.75 \mathrm{mg} / \mathrm{kg} \text {, seguido de infusión } 1.75 \mathrm{mg} / \mathrm{kg} / \mathrm{h} \text { hasta } \\
\text { terminar el procedimiento }\end{array}$ \\
\hline \multicolumn{2}{|l|}{ En Fibrinolisis } \\
\hline - Heparina No fraccionada & $\begin{array}{l}\text { Bolo IV } 60 \mathrm{U} / \mathrm{kg} \text {, máximo } 4000 \mathrm{U} \text {, seguido por infusión de } 12 \mathrm{u} / \mathrm{kg} \text {, } \\
\text { máximo } 100 \mathrm{U} / \mathrm{h} \text {, por } 24 \text { - } 48 \mathrm{~h} \text {. Mantener ACT < } 80 \mathrm{seg}\end{array}$ \\
\hline - Enoxiparina & $\begin{array}{l}\text { < } 75 \text { años: bolo } 30 \mathrm{mg} \text { IV, seguido por } 1 \mathrm{mg} / \mathrm{kg} \mathrm{SC} \text { cada } 12 \mathrm{~h} \\
\text { > } 75 \text { años: no dar bolo IV. Dosis } 0.75 \mathrm{mg} / \mathrm{kg} \mathrm{SC} \text { cada } 12 \mathrm{~h} \\
\text { Creatininemia > 2.5: administrar la dosis } 1 \mathrm{vez} \text { al día }\end{array}$ \\
\hline - Fondaparinux & Bolo $2.5 \mathrm{mg} \mathrm{IV}$, seguido de $2.5 \mathrm{mg} \mathrm{sc} 1 \mathrm{vez}$ al día \\
\hline
\end{tabular}

Es importante destacar que luego de una trombolisis exitosa los pacientes deben ser categorizados cuidadosamente, para evitar reinfartos y otras complicaciones (ver más adelante).
En pacientes con contraindicación a fibrinolisis, la indicación ideal es la angioplastía primaria. Si ello no es posible, ni siquiera tras considerar la opción de trasladar al enfermo, se puede utilizar Heparina IV, 
enoxiparina o fondaparinux, más Aspirina $500 \mathrm{mg}$ y Clopidogrel $300 \mathrm{mg}$. Hay que tener en cuenta que los pacientes no reperfundidos son los que tendrán más complicaciones, tanto en la fase aguda como en la alejada (mayor mortalidad, disfunción VI, arritmias ventriculares, aneurisma ventricular, etc). De allí que se recomienda que estos pacientes sean trasladados a un centro que cuente con posibilidades de realizar coronariografía2, 4, 5, 59. FIGURA 6.

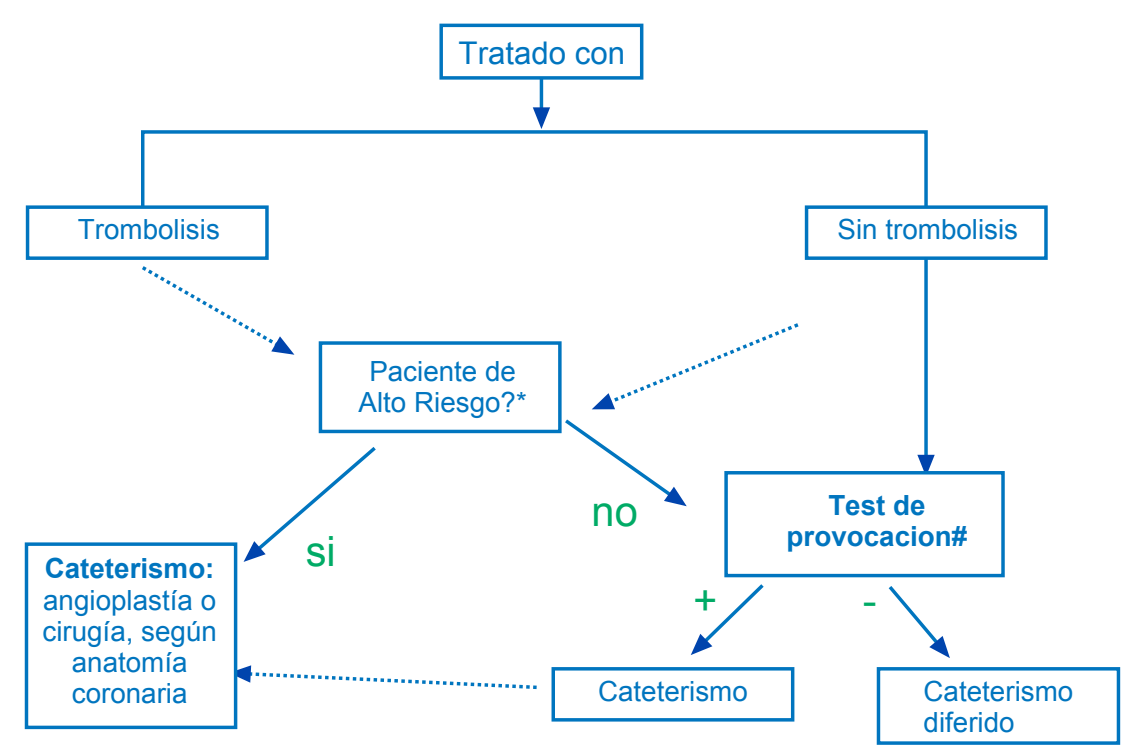

Figura 6: A quiénes realizar Coronariografía post IAM.

* Riesgo alto = IAM extenso, Killip $\geq 3$, arritmias ventriculares, hipotensión arterial, fracción de eyección < 40\%. \# = test de provocación: treadmill submáximo, sestamibi/dipiridamol, escostress dobutamina, resonancia magnética con adenosina

Clopidogrel: ver antes

\section{Dudas frecuentes en relación con el uso de Trombolíticos}

Es importante abordar brevemente algunas situaciones problemáticas que se plantean con frecuencia :

* La mortalidad de los pacientes > 75 a con un IAM es muy alta. Hasta 1/3 de los IAM ocurren en personas mayores. No existe límite de edad para la administración de agentes fibrinolíticos en el IAM. $\mathrm{Si}$ bien el riesgo asociado con el uso de estos medicamentos aumenta con la edad, también el riesgo de muerte del infarto se incrementa, de modo que el beneficio (en términos de reducción absoluta de riesgo) se mantiene60. En los pacientes añosos (> 75 años) es recomendable emplear SK en dosis más bajas, 750.000 unidades 61 .

* Los pacientes que ingresan con presión arterial $>180 / 110 \mathrm{mmHg}$ tienen mayor riesgo de hemorragia cerebral. Este riesgo persiste aún cuando la presión se logre normalizar (nitroglicerina IV, betabloqueadores). De allí que para estos casos se recomiende la angioplastía primaria. Si el IAM es extenso, el paciente es $<70$ años, y no hay disponibilidad de angioplastía, se puede asumir una trombolisis con mayor riesgo.

* No existen argumentos que sustenten el empleo de trombolíticos después de transcurridas las primeras 12 horas de infarto, salvo posiblemente en aquellos pacientes con dolor y supradesnivel ST persistente, en quienes la intensidad del dolor ha tenido fluctuaciones marcadas (indicativo por lo general de una oclusión coronaria subtotal o intermi- 
tente). En el mismo sentido, la angioplastía primaria tiene indicación en casos tardíos ( $>12$ h) si el paciente presenta hipotensión, insuficiencia cardíaca o isquemia62. Tales casos pueden corresponder a infartos no completados, isquemia en territorios vecinos al infarto, o a lesiones de múltiples vasos (ver angioplastía primaria en shock cardiogénico).

* La aparición de ondas Q en el ECG de ingreso, asociado a supradesnivel ST, no contraindica la terapia fibrinolítica en el paciente que consulta en los plazos señalados y que persiste con dolor anginoso.

\section{Revascularización mediante Cirugía de Revascularización Coronaria}

La cirugía de By Pass coronario es excepcional en la fase aguda del IAM con SDST, ya que ha sido reemplazada en la mayoría de los grandes centros por la angioplastía primaria, en razón de la reperfusión más precoz que esta técnica confiere63.

Actualmente la cirugía en el IAM con SDST está indicada como medida de rescate ante fracasos o complicaciones de la angioplastía primaria (por ejemplo: oclusión no franqueable, disección coronaria extensa, con compromiso del flujo que no pueda corregirse vía percutánea) en pacientes que presentan dolor persistente y que cursan con un IAM extenso, o presentan inestabilidad hemodinámica.

Otra indicación de cirugía coronaria en el IAM son los pacientes con anatomía coronaria de alto riesgo (ej. estenosis crítica de tronco común izquierdo), que estén dentro del período considerado más útil para la reperfusión ( $<6$ horas), aunque -por cuestión de tiempo- la angioplastía puede ser la única alternativa ante un shock cardiogénico secundario a oclusión de tronco.

La morbimortalidad operatoria en estos casos es considerablemente más alta que cuando la cirugía se realiza en forma electiva. La cirugía tiene un rol más importante en aquellos pacientes que presentan complicaciones mecánicas del IAM (ver más adelante).

\section{IAM de Ventrículo Derecho}

EI IAM de ventrículo derecho (VD) se presenta en alrededor del $10 \%$ de los IAM de pared inferior; constituye un grupo de alta mortalidad y requiere un manejo especial. En la mayoría de los casos es secundario a la oclusión de la coronaria derecha proximal. Por tratarse de un IAM de mal pronóstico, el tratamiento ideal incluye la reperfusión precoz, ya sea mediante trombolisis o angioplastía64. El diagnóstico de IAM de VD debe buscarse desde el ingreso en todo IAM de pared inferior mediante el registro ECG de las precordiales derechas (SDST $>1 \mathrm{~mm}$ en $\mathrm{V} 4 \mathrm{R}$ ). La tríada de hipotensión, ingurgitación yugular y ausencia de congestión pulmonar en la radiografía en un paciente que cursa con IAM inferior es muy característica de IAM de VD, pero poco sensible. El diagnóstico se puede confirmar ya sea con el hallazgo de SDST en V4R o por dilatación y disfunción de ventrículo derecho al ECO 2D. $\mathrm{La}$ isquemia produce distensión aguda del VD, creando un aumento de la presión intrapericárdica, lo que provoca disminución del llenado ventricular derecho, menor volumen expulsivo, caída del llenado del ventrículo izquierdo, seguido de disminución de débito sistémico, hipotensión y shock. Por lo tanto, medicamentos que disminuyen la precarga, tales como diuréticos, nitroglicerina, morfina e IECA están contraindicados en estos casos. Estos pacientes requieren de monitorización hemodinámica con Swan-Ganz y deben recibir aporte de volumen (1 - 2 litros de solución salina 9/oo en pocas horas, hasta lograr PCP de $18 \mathrm{mmHg}$ y débito cardíaco adecuado). Si a pesar de un aporte de volumen adecuado el gasto cardíaco persiste bajo, debe adicionarse dobutamina.

En muchos casos el IAM de VD se asocia a disfunción del nódulo sinusal o a bloqueo aurículo ventricular.

\section{Complicaciones en el curso de las primeras} 24 horas del infarto

A) Arritmias durante las primeras horas

Extrasistolía ventricular: los extrasístoles ventriculares 
frecuentes, polifocales, pareados o con fenómeno de «R sobre $T$ » corresponden a extrasistolía ventricular potencialmente maligna, y pueden conducir a taquicardia o fibrilación ventricular. Se tratan con lidocaína $(1 \mathrm{mg} / \mathrm{kg}$ en bolo, repitiendo la mitad de la dosis a los 15 min, seguido de infusión de 1 - $4 \mathrm{mg} / \mathrm{min})$. En cambio, el tratamiento profiláctico (en ausencia de arritmias) con lidocaína no está indicado, pues puede aumentar la mortalidad. El uso de betabloqueadores suele ser igualmente efectivo en estas arritmias, al tiempo que reducen la mortalidad 65 .

La monitorización de los enfermos y el adecuado entrenamiento del personal para realizar cardioversión eléctrica ante la eventual aparición de fibrilación ventricular han sido fundamentales para reducir la mortalidad por arritmias ventriculares graves en el curso de las primeras horas del IAM. Paralelamente, deben corregirse factores gatillantes de la extrasistolía y que pueden aumentar la inducción de taquicardia o fibrilación ventricular, como la ansiedad, hipoxemia, alteraciones electrolíticas (hipomagnesemia, hipokalemia) o del equilibrio ácido-base, frecuentes en el contexto de un IAM.

\section{Ritmo Idioventricular Acelerado (frecuencia entre}

60 y 110/min): Es por lo general autolimitado y bien tolerado; más frecuente en los IAM de pared inferior. En el contexto de trombolisis o de angioplastía primaria se le considera como una arritmia de reperfusión. Su significado pronóstico no está bien definido y por lo mismo no hay una clara indicación de tratarlos.

Taquicardia Ventricular: Salvas de taquicardia ventricular no sostenida (<30 s, y que no compromete la hemodinamia) se observan con mucha frecuencia en las primeras 24 horas y pueden ser premonitorias de fibrilación ventricular. Se tratan con lidocaína a las dosis mencionadas y con adecuada corrección de los factores gatillantes (ver comentarios previos). En casos de taquicardia ventricular sostenida (> 30 segundos) que causa hipotensión sistólica $<90 \mathrm{mmHg}$, angina o edema pulmonar, su tratamiento es la cardioversión sincronizada bajo anestesia o sedación profunda, inicialmente con 50 Joules (importante tener buena sincronización con onda R), seguida de lidocaína en la dosis señaladas. Energías más bajas, especialmente menores de 25 Joules, al igual que la aplicación de un golpe precordial -antiguamente recomendadoaparte de ser menos efectivas, podrían facilitar la inducción de fibrilación ventricular. Cuando la taquicardia ventricular es polimórfica es más probable que sea secundaria a isquemia. En esta forma de taquicardia también la lidocaina es efectiva, pero si es sostenida, generalmente es muy rápida y compromete la hemodinamia, por lo que requiere cardioversión eléctrica inmediata. A diferencia de la taquicardia monomórfica, en esta taquicardia la sincronización puede impedir la detección de los complejos ventriculares de baja amplitud, por lo que la cardioversión debe ser no sincronizada (es decir, desfibrilación) y con descargas de al menos 100 Joules. Además, debe realizarse precozmente una coronariografía.

Cuando la taquicardia ventricular es bien tolerada (generalmente con frecuencias menores a 150/min) puede intentarse la cardioversión farmacológica con lidocaína, o con Amiodarona (150 a 300 mg IV a pasar en $10 \mathrm{~min}$. seguidos de una infusión de 600 - $900 \mathrm{mg} /$ 24h), con estricto control de la presión arterial, procediéndose a la cardioversión eléctrica si no hay respuesta o si aparecen signos de hipoperfusión. Si la taquicardia es recurrente, puede aumentarse la dosis de Amiodarona, agregar betabloqueo si no existe contraindicación, o bien puede instalarse una sonda MP transitoria en ventrículo derecho, para intentar sobreestimulación eléctrica a través de un MP externo cada vez que la taquicardia recurra, y así evitar los efectos colaterales de altas dosis de antiarrítmicos. También debe tenerse presente que la causa subyacente de una arritmia ventricular recurrente o de una arritmia ventricular refractaria puede ser isquemia miocárdica; en estos casos está indicada una coronariografía, para definir la terapia más adecuada66.

Fibrilación Ventricular: La fibrilación ventricular primaria (en las primeras 48 horas) es más común en IAM extensos y se asocia a una mayor mortalidad intrahospitalaria y alejada. Su aparición puede ser precipitada por alteraciones hidroelectrolíticas, hipoxia o acidosis. El tratamiento es la desfibrilación 
eléctrica inmediata no sincronizada con descarga inicial de 200 - 300 Joules, hasta 360 Joules, en caso necesario. Las maniobras de resucitación no deben retrasar la aplicación de la descarga eléctrica. La aparición de asistolía o de disociación electromecánica post desfibrilación es de mal pronóstico. Al igual que en la taquicardia ventricular, está indicada la infusión de lidocaina por un mínimo de 24 horas. Es importante estar familiarizado con las manifestaciones de toxicidad por lidocaina (náuseas, parestesia peribucal, mareo, confusión, depresión respiratoria, bradicardia, hipotensión, convulsiones). La fibrilación ventricular de aparición más alejada (después de 2 días) es de mal pronóstico66 (ver arritmias tardías).

Fibrilación Auricular: se presenta por lo general en infartos extensos y suele ser manifestación de isquemia auricular o de distensión de la aurícula secundaria a falla ventricular. Puede desencadenar deterioro hemodinámico por respuesta ventricular rápida o por la pérdida de la contracción auricular 67,68 . El tratamiento inicial consiste en Amiodarona IV (150 a $300 \mathrm{mg}$ a pasar en 10 minutos seguida de 600 - $900 \mathrm{mg}$ en infusión durante 24 horas); conjuntamente puede administrarse Cedilanid $(0.4 \mathrm{mg}$ en bolo) para frenar la conducción AV. Con ese mismo fin en pacientes con angina sin disfunción ventricular y sin congestión pulmonar puede usarse propranolol IV, $1 \mathrm{mg}$ y luego repetir hasta $5 \mathrm{mg}$ dosis máxima, para obtener frecuencia cardíaca $<70$ por minuto, para evitar el aumento del consumo de oxígeno miocárdico que provocan las frecuencias altas. En pacientes con severo compromiso hemodinámico se debe proceder a realizar cardioversión sincronizada inmediata (100 - 200 Joules).

La fibrilación auricular se asocia a mayor riesgo de embolía sistémica, por lo cual debe usarse heparina IV por algunos días. Los pacientes con fibrilación auricular persistente deben recibir anticoagulantes orales en forma permanente.

\section{Bradiarritmias}

Son más comunes en los IAM de pared inferior. Bradicardia sinusal: es un hallazgo habitual en los infartos de pared inferior. Cuando la frecuencia cardíaca baja de $40 / \mathrm{min}$ o si el paciente presenta hipotensión o signos de hipoperfusión, está indicado el tratamiento con Atropina ( 0.5 a $1 \mathrm{mg}$ IV en bolo, y repetir de acuerdo a la respuesta). Puede requerirse la inserción de una sonda MP para estimular el ventrículo derecho o, idealmente, la aurícula derecha en los raros casos que no responden a esta droga.

Bloqueo Auriculo-ventricular (BAV) de primer grado : Es frecuente y no tiene implicancias pronósticas salvo en el contexto de un infarto agudo de pared anterior con bloqueo bifascicular (en quienes el sitio del bloqueo es hisiano más que nodal). No requiere de tratamiento específico.

\section{Bloqueo auriculo-ventricular de segundo grado tipo} Mobitz I (Wenckebach): es generalmente manifestación de isquemia nodal, al igual que en el caso del BAV simple. De buen pronóstico y generalmente transitorio (24 a 72 horas), suele responder a atropina IV. Ver más abajo.

Bloqueo aurículo-ventricular de segundo grado tipo Mobitz II: se asocia con bloqueos de la conducción intraventricular e infartos de pared inferior o extensos de pared anterior. Progresa frecuentemente al BAV completo y es indicación de marcapaso transitorio.

Bloqueo aurículo-ventricular completo o de tercer grado: se presenta en 5 a 10\% de los IAM. Es mucho más frecuente en los infartos de pared inferior, pero también puede ocurrir en los IAM de pared anterior. Cuando ocurre en el curso de un IAM de pared anterior, son precedidos de bloqueo completo de rama derecha o izquierda, y la aparición del BAVC es brusca. Estos casos constituyen un subgrupo de muy mal pronóstico (70 a $80 \%$ de mortalidad intrahospitalaria), pues implican compromiso isquémico muy extenso.

Por lo general, los BAVC en IAM de pared inferior tienen buen pronóstico, y regresan antes de 7 días. Se relacionan con oclusiones coronarias ubicadas antes de la rama aurículo ventricular. 
E. Guarda , J. C. Prieto, P. Sanhueza, C. Dauvergne, R. Asenjo, R. Corbalán.

EI BAVC es indicación de marcapaso transitorio inmediato.

La administración intravenosa de atropina (especialmente en presencia de ritmo de escape con QRS angosto) o el empleo de marcapasos externos pueden estabilizar al paciente con bradicardias extremas mientras se instala la sonda de marcapaso transitoria por vía venosa (subclavia o yugular) bajo visión radioscópica. Si no se dispone de fluoroscopía, son útiles los cables marcapasos con balón.

Bloqueos de la Conducción Intraventricular: La aparición de un nuevo bloqueo completo de rama izquierda o de un bloqueo bifascicular en el curso de un IAM implica compromiso septal proximal (generalmente por oclusión de la arteria descendente anterior proximal) y constituye indicación de marcapaso transitorio por el riesgo de progresión al BAV completo a nivel hisiano con asistolía. Aún con el uso de marcapasos, la mortalidad de estos pacientes es muy elevada a causa del extenso compromiso miocárdico asociado69. En los casos de nuevo BCRI en IAM de pared inferior, esto se observa en pacientes con enfermedad coronaria difusa y mala función ventricular, por lo que también tienen un mal pronóstico.

En el paciente con IAM de pared anterior con BCRI o bloqueo bifascicular y sin ECG previo, surge la duda respecto a la necesidad de instalar una sonda de marcapasos. En general, en pacientes jóvenes (en quienes la prevalencia de BCRI es baja) con imagen Eco 2D de aquinesia de todo el septum, la probabilidad de un bloqueo agudo (que requiere de marcapasos) es más alta que en el paciente mayor sin compromiso del septum proximal por ecocardiografía. En el caso de no contar con Eco 2D, y no tener clara la existencia previa de un bloqueo de rama, es razonable instalar una sonda MP preventiva, o al menos conectar al paciente a un MP externo transtorácico si está disponible. Las consecuencias de una asistolía en un paciente de esta gravedad son mayores que los riesgos de instalar un sistema de estimulación transitorio. Las indicaciones de MP transitorio en el IAM se presentan en la tabla 370 .

Tabla 3: indicaciones de marcapado transitorio en el IAM

\begin{tabular}{|l|}
\hline$*$ Bloqueo aurículo-ventricular completo \\
\hline * Asistolía \\
\hline * Aparición de bloqueo bifascicular (BCRD + HBI o HBIP), o de BCRI - BAV I grado \\
\hline * BAV tipo Mobitz II \\
\hline * Bloqueo aurículo-ventricular de segundo grado Mobitz I con hipotensión que no \\
responde a atropina. \\
\hline * Bradicardoa sintomática de cualquier tipo que no responda a la atropina \\
\hline * Manejo de la taquicardia ventricular refractaria \\
\hline
\end{tabular}

\section{B) Complicaciones mecánicas del IAM}

Ruptura de pared libre: Es la más frecuente de las complicaciones mecánicas. Aparece generalmente entre el segundo y el séptimo día de evolución y da cuenta de al menos un $15 \%$ de las muertes intrahospitalarias del IAM. Se presenta generalmente como muerte súbita por taponamiento, con disociación electromecánica, sin dar tiempo para una reparación quirúrgica. Es más frecuente en pa- cientes mayores, con antecedentes de HTA, cursando un primer infarto. En algunos casos, la ruptura ocurre más lentamente, por un trombo que ocluye la perforación o por adherencias al pericardio (ruptura encubierta o subaguda), lo que da tiempo para la cirugía de rescate. Los casos de ruptura encubierta pueden sospecharse en pacientes que presentan dolor persistente o reaparición del dolor y deterioro hemodinámico transitorio con o sin alteraciones 
del ECG. Ante la sospecha, debe realizarse un ecocardiograma y proceder a cirugía inmediata si se confirma hemopericardio.

Ruptura de septum interventricular: Se observa en aproximadamente $2 \%$ de los pacientes, especialmente en IAM extensos, tanto de pared anterior como inferior (siendo más frecuentes en los primeros, pero de peor pronóstico en los segundos). Debe sospecharse en pacientes que presentan un brusco deterioro hemodinámico asociado a la aparición de un soplo holosistólico paraesternal izquierdo bajo. El diagnóstico se confirma por ecocardiografía con Doppler color, que muestra una zona de adelgazamiento y aquinesia septal, frecuentemente con flujo transeptal visible mediante mapeo Doppler color. El salto oximétrico a nivel del ventrículo derecho durante la inserción de un cateter de Swan Ganz permite confirmar y cuantificar el cortocircuito de izquierda a derecha a nivel ventricular. Se debe implantar un balón de contrapulsación intraaórtico para estabilizar al paciente mientras se espera la cirugía, la cual debiera efectuarse precozmente, pues aunque el paciente esté estable, el orificio puede aumentar súbitamente ${ }^{71}$. El mejor momento para la cirugía de esta condición es aún materia de debate.

Insuficiencia mitral aguda: Suele aparecer entre los días 2 y 7 . Puede deberse a dilatación aguda del anillo mitral, secundario a dilatación ventricular, o a compromiso isquémico del músculo papilar. Si bien la disfunción transitoria del músculo papilar se observa con frecuencia en infartos de pared inferior y lateral, la ruptura del músculo se presenta en menos del $1 \%$ de los IAM (generalmente por compromiso del músculo postero-medial, por oclusión de una rama circunfleja). A diferencia de los pacientes con ruptura septal, el infarto puede ser pequeño (incluso no transmural). El diagnóstico se basa en la aparición de congestión pulmonar e hipotensión y de un soplo holosistólico de intensidad variable (muchas veces inaudible, particularmente en pacientes con severo compromiso hemodinámico y congestión pulmonar masiva). En el Eco Doppler puede obser- varse un ventrículo izquierdo hiperdinámico, un velo mitral disfuncionante (con «jet» de regurgitación excéntrico) y, ocasionalmente, la evidencia de un trozo de músculo papilar desinsertado con sus respectivas cuerdas flotando en el interior de la cavidad. Lo más frecuente, sin embargo, es que sólo exista disfunción y no ruptura del músculo papilar. La curva del capilar pulmonar mostrará onda «V» importante 72 .

El paciente debe ser trasladado de inmediato a un centro con cirugía cardíaca. La mortalidad es > de $80 \%$ con terapia médica, y con cirugía esta cifra puede reducirse significativamente. Los mejores resultados se obtienen cuando la cirugía se realiza tempranamente, con pocas horas de evolución del shock cardiogénico. Mientras se prepara la operación, se debe intentar estabilizar al paciente mediante el uso de inótropos, vasodilatadores como el nitroprusiato y balón de contrapulsación. Ocasionalmente, con estas medidas se consigue estabilidad hemodinámica suficiente como para realizar coronariografía previo a la cirugía, con el fin de practicar la revascularización apropiada más la corrección pertinente (reemplazo valvular, la mayor parte de las veces $)^{73}$.

\section{C) Complicaciones Hemodinámicas}

Estas han disminuido con las terapias de reperfusión precoz. El cese de la perfusión coronaria se traduce casi instántaneamente en disfunción ventricular diastólica (disminución de la distensibilidad) y sistólica (disminución de la contractilidad). La combinación de ambas se manifiesta clínicamente como congestión pulmonar, hipotensión o hipodébito. Los objetivos terapéuticos en un paciente con compromiso hemodinámico son:

* Mantener una adecuada presión de perfusión coronaria ( presión arterial media > $70 \mathrm{mmHg}$ ), a fin de limitar la extensión de la necrosis/isquemia.

* Obtener un Indice Cardíaco > $2.2 \mathrm{lt} / \mathrm{min}$

* Obtener una precarga (presión de fin de diástole del ventrículo izquierdo = presión de capilar pulmonar, PCP) adecuada para un ventrículo poco distensible, lo cual en general significan $\mathrm{PCP}=15-18 \mathrm{mmHg}$.

* Mantener una postcarga baja (definida en tér- 
E. Guarda , J. C. Prieto, P. Sanhueza, C. Dauvergne, R. Asenjo, R. Corbalán.

minos de resistencia vascular sistémica= RVS), que facilite el vaciamiento ventricular y contribuya a una adecuada perfusión tisular.

A continuación, se analiza el manejo de determinadas situaciones hemodinámicas frecuentes de observar en el IAM.

\section{Hipotensión arterial sin signos congestivos pulmonares:}

Situación frecuente en las primeras horas del IAM, ya sea por hipovolemia real (dolor, sudoración, vómitos) o relativa (presión de llenado insuficiente para un ventrículo que se ha tornado poco distensible). En estos casos, la Rx de Tórax es fundamental para comprobar la ausencia de congestión pulmonar. En infartos de pared inferior, la hipotensión suele asociarse con bradicardia, como manifestación vasovagal. El tratamiento consiste en:

* Aumento rápido de la precarga (elevar extremidades inferiores, infusión de suero fisiológico en cargas de $100 \mathrm{ml}$ cada $10 \mathrm{~min}$, observando presión arterial y aparición de crépitos pulmonares).

* Si se sospecha una reacción vagal, la administración de Atropina ( 0.5 a $1 \mathrm{mg}$ IV) es efectiva. En el paciente con infarto de pared inferior refractario a estas maniobras, debe sospecharse el compromiso de ventrículo derecho (ver más arriba).

\section{Congestión pulmonar sin hipotensión arterial:}

En su forma leve, se presenta como disnea con presencia de crépitos en ambas bases. En su forma severa, se manifiesta como edema agudo del pulmón, el que generalmente se asocia con marcada vasoconstricción cutánea y oliguria (hipodébito). El tratamiento consiste en:

- Asegurar una adecuada oxigenación (uso de bigoteras, mascarillas o ventilación mécanica en caso de desaturación refractaria).

- Reducir la pre y post carga, mediante diuréticos (por lo general Furosemida en bolos de $10 \mathrm{mg} \mathrm{IV}$ ), morfina 2-3 mg IV, y Nitroglicerina en infusión intravenosa, iniciando la infusión con $10 \mathrm{ug} / \mathrm{min}$. En el paciente hipertenso, el uso de Nitroglicerina es preferible por la ventaja hemodinámica adicional que confiere la reducción de la RVS y la vasodilatación del territorio coronario. Debe evitarse la hipotensión arterial y la taquicardia compensatoria. El Nitroprusiato de Sodio es muy efectivo como vasodilatador arterial y venoso, pero su uso es más complicado por la necesidad de contar con línea de presión arterial. En el paciente con congestión pulmonar severa, el monitoreo hemodinámico con catéter de Swan Ganz permite ajustar la precarga (y así optimizar el débito) con mucho mayor precisión que lo que se obtiene con la auscultación pulmonar y radiografía de tórax. Ver tabla 4.

Tabla 4: Indicaciones de Monitorización Hemodinámica (Swan-Ganz) en el IAM (no debe retrasar la terapia de reperfusión precoz)

\begin{tabular}{|l|}
\hline * Edema pulmonar agudo que no responde al tratamiento \\
\hline * Insuficiencia cardíaca severa o progresiva \\
\hline * Shock cardiogénico \\
\hline * Sospecha de complicacione mecánicas: rotura septum interventricular \\
o de músculo papilar \\
* Hipotensión refractaria al aporte de volumen \\
\hline
\end{tabular}

\section{D) Shock Cardiogénico}

Representa la forma extrema de falla ventricular izquierda y se asocia con una mortalidad muy elevada en pacientes no reperfundidos. De allí que la mejor terapia del shock cardiogénico sea evitar su aparición mediante una reperfusión precoz ${ }^{74}$.
El shock cardiogénico primario (paciente que ingresa en shock o lo presenta en las primeras horas de evolución) se asocia a una mortalidad superior al $80 \%$. Los pacientes que desarrollan shock cardiogénico dentro de las primeras 24 - 48 horas pueden tener un pronóstico menos ominoso. 
En los casos de shock cardiogénico es muy difícil implementar trombolisis sistémica por la hipotensión arterial y compromiso general que presentan los pacientes, y el tratamiento médico exclusivo implicará una mortalidad muy elevada ( $>80 \%$ ). Por ello un paciente con IAM y shock cardiogénico debe ser trasladado rápidamente a un centro asistencial donde se pueda efectuar una angiografía coronaria de urgencia con apoyo de balón intraórtico. Tanto la angioplastía primaria de rescate o la cirugía coronaria de urgencia han probado ser eficaces en reducir la mortalidad del shock cardiogénico a cifras cercanas al $50 \%$. La revascularización en esos casos suele implicar tratamiento de varias arterias, no sólo la culpable 75 .

Las medidas de tratamiento médico a implementar en el shock cardiogénico son las siguientes:

- Mantener ritmo sinusal.

- Asegurar una oxigenación adecuada, lo cual corrientemente implica ventilación mecánica. Aunque es posible mantener saturaciones de oxígeno aceptable mediante mascarillas de recirculación con fracción inspirada de oxígeno de $70-100 \%$, el trabajo respiratorio que ello demanda puede complicar aún más la evolución del paciente.

- Corrección de alteraciones hidroelectrolíticas y ácidobase, especialmente la acidosis láctica.

- Mantener una presión arterial media sobre $70 \mathrm{~mm} \mathrm{Hg}$. Ello requiere generalmente del uso de Dopamina (en dosis de 1 a $20 \mu \mathrm{g} / \mathrm{kg} / \mathrm{min}$ en infusión intravenosa) y ocasionalmente, en el paciente profundamente hipotenso, de Norepinefrina.

- Optimizar el débito cardíaco agregando inótropos con acción vasodilatadora como la Dobutamina (1 a $15 \mu \mathrm{g} / \mathrm{kg} / \mathrm{min}$ en infusión intravenosa, incrementando la dosis dependiendo del balance entre aumento del gasto cardíaco por un lado, versus taquicardia o hipotensión). La Milrinona, droga inhibidora de la fosfodiesterasa, es útil en pacientes en quienes la catecolaminas exacerban las arritmias ventriculares. La milrinona se usa en dosis de 0.25 hasta $1 \mu \mathrm{g} / \mathrm{kg} / \mathrm{min}$, con o sin bolo inicial de $50 \mu \mathrm{g} / \mathrm{kg}$ (el bolo puede inducir hipotensión).

- Optimizar precarga llevando la PCP a valores no inferiores a $20 \mathrm{mmHg}$. En ocasiones esto provoca congestión pulmonar, y eventualmente obliga a conectar al paciente a ventilación mecánica. Se requiere manejo cuidadoso en esta situación, balanceando precarga, gasto cardíaco, congestión pulmonar, uso de presión positiva al final de la espiración, etc.

- Post carga: los vasodilatadores están contraindicados en el shock cardiogénico. Su empleo cauteloso puede considerarse sólo en pacientes con falla ventricular secundaria a insuficiencia mitral severa o ruptura de septum (ver abajo).

- Balón de contrapulsación intra aórtico: El balón de contrapulsación aórtico permite reducir la postcarga a la vez que eleva la presión de perfusión coronaria durante la diástole. Es de gran utilidad en el infarto con shock cardiogénico o para el manejo de pacientes con isquemia post IAM refractaria a terapia médica, mientras se implementan maniobras de reperfusión coronaria (ej. Angioplastía primaria o de rescate). Su colocación también está indicada en casos de ruptura del septum interventricular o de músculo papilar, como medida de apoyo peri-operatorio. El balón de contrapulsación por sí solo no mejora la mortalidad, pero ayuda a estabilizar la hemodinamia de estos pacientes críticos, permitiendo realizar coronariografía y procedimientos de revascularización en mejores condiciones.

\section{Fase hospitalaria tardia y Evaluación del paciente previo al alta}

Monitoreo ECG: se mantiene, por lo general, durante un mínimo de 48 a 72 horas en el paciente no complicado, por cuanto este es el período en que se observa la mayor incidencia de arritmias ventriculares complejas, incluidas la fibrilación ventricular. La vigilancia monitorizada debe prolongarse en casos de infartos extensos, inestabilidad hemodinámica, isquemia recurrente o arritmias persistentes.

Reposo: El valor del reposo en cama en el paciente con IAM de curso no complicado es incierto y la mayor parte de los pacientes en esta categoría pueden 
reiniciar actividad física liviana (caminar en la pieza, ir al baño ) a las 48 - 72 horas del evento 76 .

La duración de la hospitalización depende del curso clínico del infarto, de la edad del paciente y de la accesibilidad a centros asistenciales en caso necesario. La permanencia habitual de pacientes con infartos no complicados es de 4 días.

a) Educación del paciente y control de factores de riesgo: Debe aprovecharse la convalescencia (período de gran motivación) para indentificar los factores de riesgo del paciente e iniciar un programa de prevención secundaria. TABLA $577,78$.

Tabla 5: Objetivos en prevencion Secundaria post IAM

\begin{tabular}{|l|l|}
\hline $\begin{array}{l}\text { Tabaco } \\
\text { Objetivo: suspensión completa }\end{array}$ & $\begin{array}{l}\text { Suspensión del hábito y evitar el humo de terceros. Apoyo psicológico y } \\
\text { medicamentos: ansiolíticos, parches de nicotina, bupropion, vareniclina }\end{array}$ \\
\hline $\begin{array}{l}\text { Presión Arterial } \\
\text { Objetivo: PA }<130 / 80 \mathrm{mmHg}\end{array}$ & $\begin{array}{l}\text { Estilo de vida saludable, ejercicio físico, restricción de sal y de la ingesta de alcohol, } \\
\text { mayor consumo de frutas y verduras; IECA (o ARA II), betabloqueadores }\end{array}$ \\
\hline $\begin{array}{l}\text { Dislipidemia } \\
\text { Objetivo: } \mathrm{LDL}<100 \text { (idealmente } \\
<70) \mathrm{mg} / \mathrm{dl}, \mathrm{TG}<150 \mathrm{mg} / \mathrm{dl}\end{array}$ & $\begin{array}{l}\text { Estilo de vida saludable, restricción de grasas saturadas, apoyo nutricional, ejercicio } \\
\text { físico reglar. Usar estatinas potentes (atorvastatina, rosuvastatina) en dosis adecuadas } \\
\text { para conseguir los objetivos. Agregar fibratos si TG persisten altos. }\end{array}$ \\
\hline $\begin{array}{l}\text { Actividad física } \\
\text { Objetivo: } 30 \mathrm{~min} 5 \mathrm{veces} \text { por } \\
\text { semana }\end{array}$ & $\begin{array}{l}\text { Idealmente en programa de rehabilitación (supervisado), guiado por test de esfuerzo. } \\
\text { Asociar a caminatas, trote, bicicleta. }\end{array}$ \\
\hline $\begin{array}{l}\text { Peso } \\
\text { Objetivo: IMC }<25 \mathrm{Kg} / \mathrm{m} 2\end{array}$ & $\begin{array}{l}\text { Restricción calórica. Aumentar actividad física, Consejo y evaluaciones nutricionales } \\
\text { periódicas }\end{array}$ \\
\hline $\begin{array}{l}\text { Diabetes Mellitus } \\
\text { Objetivo: HbA1c }<7 \%\end{array}$ & Dieta estricta, uso de hipoglicemiantes en dosis adecuadas, ejercicio físico \\
\hline Medicamentos & $\begin{array}{l}\text { Aspirina (de por vida) y clopidrogrel, este último por plazos variables dependiendo del } \\
\text { stent utilizado; IECA a todos quienes no tengan contraindicaciones (o ARA II); }\end{array}$ \\
& Espironolactona si FE < 40\%. Betabloqueadores, con las restricciones habituales \\
\hline
\end{tabular}

Hipertensión arterial: El control de la presión arterial debe lograrse con dieta, restricción de sal y medicamentos; especialmente indicados son los IECA, ARAll y los betabloqueadores, pero con frecuencia es necesario agregar diuréticos $y$ dihidropiridinas de acción prolongada (amlodipino, felodipino) para alcanzar las cifras recomendadas.

Tabaquismo: Los fumadores tienen el doble de riesgo de presentar un sindrome coronario agudo que sus pares no fumadores. Aquellos que dejan de fumar después de un IAM reducen en un $30 \%$ el riesgo de muerte y reinfarto tardío. Así, la suspensión del hábito es la más potente de las indicaciones preventivas. Aparte de educación dirigida, y de obtener el apoyo familiar, varios medicamentos puede ayudar a evitar la recidiva, tales como parches de nicotina, bupropion, vareniclina y otros antidepresivos. Puede comenzarse con ellos en el período intrahospitalario, con adecuada supervisión 79 .

Dislipidemia: La determinación de los niveles plasmáticos de colesterol debe hacerse al ingreso del paciente, antes de que el stress y los medicamentos alteren el perfil lipídico. Independiente de lo anterior, las guías internacionales recomiendan el uso de estatinas potentes en todo paciente que ingrese con un IAM, y reducir las dosis en controles ambulatorios de acuerdo a los valores que se se obtengan a las 6 - 8 semanas. El colesterol LDL debiera ser mantenido en valores tan bajos como $70-80 \mathrm{mg} / \mathrm{dl}$ en esta población de alto riesgo3,5.

Aspirina: Su administración a permanencia se sustenta en los resultados de varios estudios randomizados con grupo control que revelan un $9 \mathrm{y}$ 
$20 \%$ de reducción en mortalidad y tasa de reinfarto, respectivamente, en sobrevivientes de un IAM. Las dosis más utilizadas fluctúan entre los 100 y 325 mg diarios.

Betabloqueadores: su uso rutinario en sobrevivientes de IAM está avalado por más de 20 estudios randomizados, realizados con diversos beta bloqueadores. Se ha observado una reducción de la mortalidad de aproximadamente $20 \%$, con una reducción de $30 \%$ de incidencia de muerte súbita (atribuible a arritmias o reinfarto). De allí que todo paciente con IAM debe recibir betabloqueadores a menos que exista una contraindicación formal. Estos medicamentos deben iniciarse cuando el paciente está estable25,26. Al respecto cabe destacar 3 puntos:

- Los pacientes con función ventricular deteriorada (fracción de eyección ventricular izquierda inferior a $40 \%$ ), en quienes los betabloqueadores suelen evitarse por el temor de acentuar los síntomas de congestión, son precisamente el subgrupo que más se beneficia en términos de reducción de mortalidad. Los betabloqueadores también están indicados en ancianos y en diabéticos.

- Las dosis de estos medicamentos deben incrementarse en forma gradual para obtener un beta bloqueo efectivo (frecuencia cardíaca alrededor de 60 latidos por minuto en reposo), y sin descompensar al paciente.

- Los beta bloqueadores con mejor evidencia a largo plazo en cardiopatía isquémica son carvedilol, bisoprolol y metoprolol. El atenolol, en cambio, tiene menos evidencias a favor.

Antagonistas del calcio: Al igual que los nitratos, tampoco tienen indicación en la prevención secundaria del paciente infartado. Su administración está contraindicada en sujetos con congestión pulmonar o fracción de eyección ventricular izquierda inferior a $40 \% 80$. Se puede indicar dihidropiridinas de acción prolongada (amlodipino, felodipino) en casos de hipertensión arterial resistente a IECA una vez que el paciente está estable, y que esté recibiendo betabloqueadores, para evitar una taquicardia refleja que pueda gatillar arritmias.
Inhibidores de la enzima convertidora de angiotensina (IECA): Estos medicamentos han demostrado aumentar la sobrevida de los pacientes con IAM que cursan con disfunción VI (fracción de eyección $<40 \%$ ) o que presentan signos de ICC81-83. Se recomienda que la administración sea precoz (2do o 3er día), una vez que la presión arterial y la volemia se hayan estabilizado. Se administran en dosis crecientes, evitando provocar hipotensión arterial o falla renal. Sus efecto favorables, sin embargo, se centran claramente en los pacientes con infarto anterior, o con infartos previos, con mala función ventricular basal, insuficiencia cardíaca o taquicardia. Los IECA favorecen un mejor remodelamiento miocárdico, previniendo de esta manera complicaciones alejadas (reinfarto, Insuficiencia cardíaca, muerte). Las principales contraindicaciones de los IECA son la presión arterial $<100 \mathrm{mmHg}$, insuficiencia renal, estenosis bilateral de arterias renales, alergia previa a este tipo de fármacos.

En caso de intolerancia o contraindicación a los IECA puede considerarse el empleo de antagonistas del receptor 2 de la angiotensina II (ARAII)84,85. Pueden combinarse con antagonistas de la aldosterona como eplerenona o espironolactona. Vigilar la aparición de hiperkalemia.

Antagonistas de aldosterona: El Estudio Ephesus (86) demostró beneficio de la eplerenona (antialdosterónico sin efecto antiandrogénico) en IAM con falla ventricular izquierda. La espironolactona es el antialdosterónico que más se emplea en nuestro país. Debe vigilarse la aparición de hiperkalemia, favorecida también por los IECA.

b ) Isquemia recurrente y reinfarto

El diagnóstico se basa en la reaparición de angina (no siempre presente) y/o depresión o elevación del segmento ST de más de $1 \mathrm{~mm}$. Ambas condiciones pueden manifestarse clínicamente por taquicardia sinusal, deterioro hemodinámico, congestión pulmonar, aparición de arritmias ventriculares complejas. Ante la recurrencia de síntomas, debe realizarse una nueva serie de marcadores de necrosis miocárdica. Por su vida media corta (empiezan a caer a las 24 horas post IAM), la medición de las CK-MB son la primera 
elección en este contexto. En cambio, como las troponinas se mantienen elevadas hasta por 2 semanas post IAM, no permiten precisar si hay reinfarto en ese plazo o se trata sólo de la elevación secundaria al evento inicial.
El tratamiento de la isquemia post IAM implica la administración de heparina IV, nitroglicerina IV, betabloqueadores y una coronariografía de urgencia, aunque ello signifique trasladar al paciente a un centro que cuente con cardiología intervencional. figura 7.

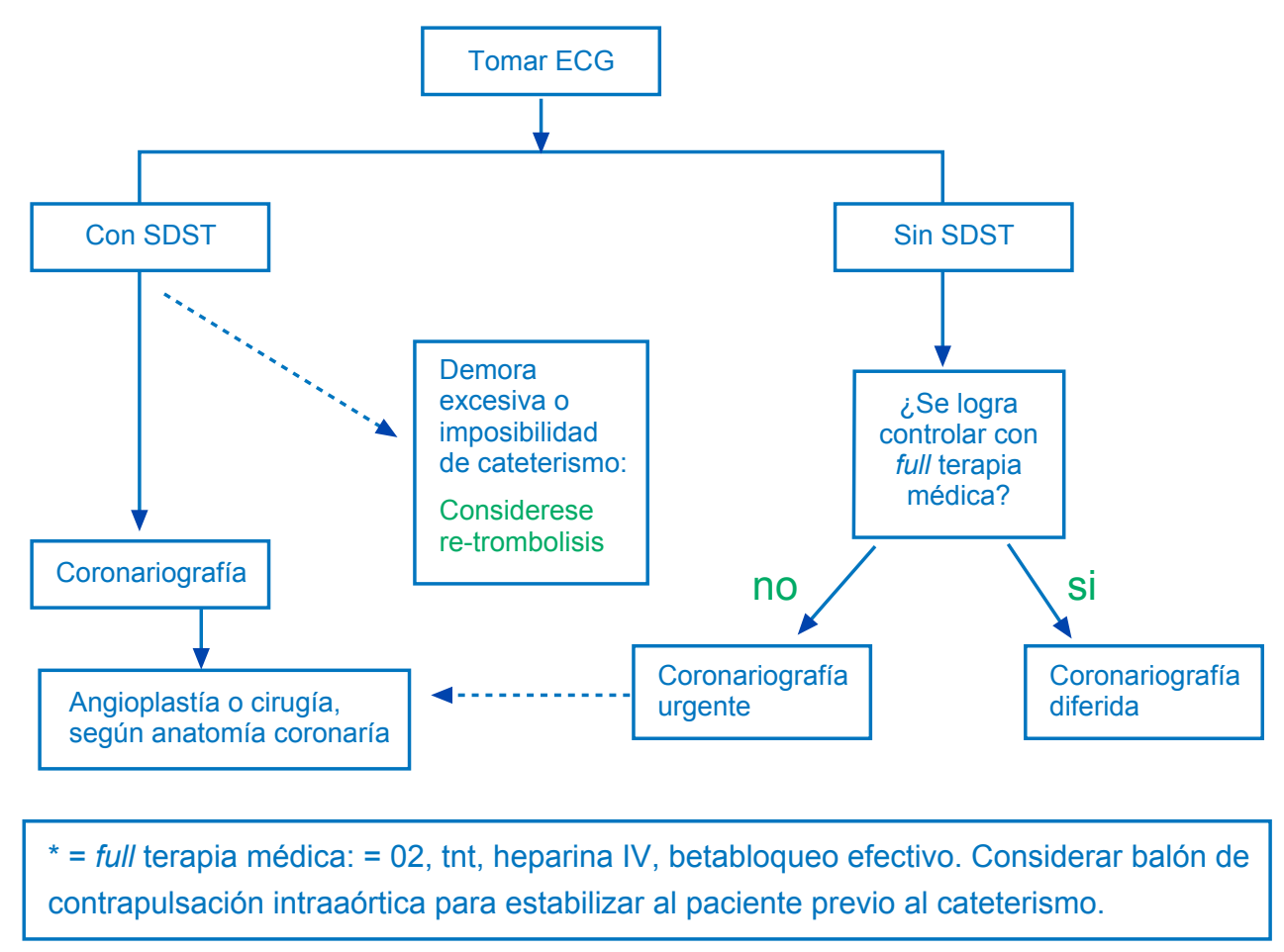

Figura 7: Conducta frente Angina o Isquemia post IAM con SDST

El tratamiento del reinfarto va dirigido a la reperfusión, idealmente mediante angioplastía. En pacientes tratados con SK puede repetirse la dosis pasadas las primeras 72 horas y dentro de los primeros 7 días del infarto. La SK induce la formación de anticuerpos que impiden su reutilización por lo menos por 1 año. En su defecto pueden emplearse otros trombolíticos (t-PA-reteplase, TNKtenecteplase) sin la limitación impuesta por la antigenicidad de la SK.

Arritmias ventriculares tardías: Ver más adelante.

c) Otras complicaciones del período hospitalario

- Trombosis venosa profunda y tromboembolismo pulmonar. Menos frecuentes que en décadas anteriores, por el uso habitual de heparina profiláctica. Para la profilaxis debe usarse idealmente heparina de bajo peso molecular, especialmente en casos de insuficiencia cardíaca importante, ventilación mecánica, etc. Si se pesquisa embolía pulmonar, debe tratarse con heparina no fraccionada IV, seguida de anticoagulantes orales por mínimo 6 meses.

- Trombo intraventricular: Debe sospecharse especialmente en IAM anterior extenso, y confirmarse con ecocardiograma transtorácico. Debe tratarse de manera semejante a lo señalado en el párrafo anterior.

- Pericarditis: Puede aparecer precozmente después del IAM, manifestándose por dolor precordial. EI dolor puede ser intenso, y ser confundido con angina post IAM. Su principal característica es que aumenta con los movimientos respiratorios, y tiende 
a disminuir al sentar al paciente en su cama y pedirle que acerque la cabeza a los pies. Los frotes pericárdicos son muy específicos, pero no son frecuentes de auscultar. EI ECG suele mostrar SDST difuso (en todas las derivaciones), y sin cambios recíprocos. La ecografía puede mostrar diversa cantidad de líquido en la cavidad pericárdica, y puede pesquisar colapso de cavidades derechas, en casos de derrames masivos y de instalación rápida, los cuales pueden llevar a taponamiento cardíaco. En tales casos corresponde efectuar una pericardiocentesis de urgencia. Los casos menos graves se tratan con AINES. Debe evitarse el uso de heparina ante pericarditis importantes, por el riesgo de hemopericardio.

\section{Evaluación previa al alta}

Como se ha señalado, el paciente que es dado de alta luego de un IAM enfrenta un riesgo de mortalidad cardiovascular y de reinfarto de aproximadamente 6 y $5 \%$, respectivamente, durante el primer año. El riesgo de muerte post IAM guarda relación fundamentalmente con tres factores pronóstico: función ventricular izquierda residual, isquemia residual y detección de arritmias ventriculares complejas. Estos factores tienen que ser analizados previo al alta:

a) función ventricular izquierda residual post infarto: La evaluación de la función ventricular puede ser clínica (anamnesis, ex. físico) y mediante exámenes como la ecocardiografía o la ventriculografía radioisotópica. La presencia de insuficiencia cardíaca congestiva o de fracción de eyección $<40 \%$ post IAM se asocia con una sobrevida inferior del $50 \%$ a 5 años. La evaluación pronóstica debe realizarse semanas después del evento, especialmente en los pacientes reperfundidos, debido a la lenta recuperación del músculo "atontado", aquel que estuvo sometido a isquemia intensa, y que se recupera desde el momento en que se reestableció el flujo.

Las siguientes intervenciones mejoran el pronóstico de estos pacientes:
- Uso de IECA (o de ARAII)

- Betabloqueadores (no siempre tolerados)

- Antagonistas de la aldosterona

- Tratamiento de isquemia asociada mediante revascularización. Este aspecto es muy importante, debido a que con frecuencia pueden existir zonas con músculo "hibernado". Se denomina de esa manera a músculo que está disfuncionante por isquemia intermitente, repetida, pero que puede recuperar su funcionalidad cuando se revasculariza. La función contráctil puede mejorar significativamente.

b) isquemia residual y extensión de la enfermedad coronaria: Los pacientes con evolución no complicada pueden ser evaluados con prueba de esfuerzo. Esta puede efectuarse precozmente (previo al alta), con un test submáximo o, de preferencia, mediante una prueba máxima (limitada por síntomas) a partir de la tercera semana post IAM. Se recomienda no suspender los betabloqueadores previo al test (que no tiene una finalidad diagnóstica sino de evaluación pronóstica). Los pacientes capaces de completar la tercera fase del protocolo de Bruce sin manifestaciones de isquemia o aparición de arritmias ventriculares complejas tienen un excelente pronóstico $(<2 \%$ de mortalidad al año) y no requieren de mayor estudio. Esto es válido tanto para pacientes sometidos a tratamientos de reperfusión como para aquellos que no lo recibieron.

En cambio, la presencia de isquemia inducible se asocia con un mayor riesgo de reinfarto y muerte. De allí que en estos casos sea aconsejable efectuar una coronariografía para definir la necesidad de revascularización. Aquellos sujetos con ECG no interpretable o incapaces de ejercitarse pueden evaluarse mediante técnicas radioisotópicas, ecocardiografía de estrés o, directamente, angiografía, según la disponibilidad y experiencia de cada centro.

Los pacientes tratados con angioplastía primaria tienen -obviamente- una anatomía coronaria conocida. Sin embargo, con frecuencia emergen dudas respecto de si es necesario revascularizar 
otros territorios, con lesiones intermedias. Un test de isquemia puede ayudar a decidir la conducta más adecuada en esos casos. Figura 6 3-5. Es fundamental que los pacientes que recibieron trombolisis y -más aún- quienes no la recibieron, sean incorporados a un protocolo que permita pesquisar isquemia residual. Las complicaciones isquémicas se pueden prevenir por medio de revascularización, de modo que deben crearse instancias para que todos esos pacientes sean evaluados adecuadamente en los días o semanas post IAM.

c) arritmias ventriculares complejas después de 48 hrs post IAM: Se pueden distinguir las arritmias ventriculares complejas, la taquicardia ventricular (TV) sostenida y la fibrilación ventricular. Estas 2 últimas entidades son netamente malignas; la TV sostenida suele preceder a la FV, por lo cual debe ser tratada agresivamente. Las arritmias ventriculares complejas (salvas de TV autolimitadas, EV frecuente y polimórficas), son pesquisadas en el monitor, o en un Holter de $24 \mathrm{hrs}, \mathrm{y}$ son predictoras de muerte súbita, especialmente si se asocian a evidencias de disfuncion VI. Se deben corregir eventualmente mediante el uso de betabloqueadores y al sustrato isquémico mediante revascularización. El uso de antiarrítmicos tipo IA (quinidina, procainamida), IC (flecainide y encainide), y el tipo III D-Sotalol, se asocian a mayor mortalidad en pacientes con disfunción ventricular post infarto del miocardio, por lo que están formalmente contraindicados. La amiodarona, antiarrítmico tipo III ampliamente usado en Chile, disminuye la incidencia de muerte súbita (MS), con un efecto neutro sobre la mortalidad global en estos enfermos. También puede usarse en pacientes con fracción de eyección baja post IAM, porque aunque no mejora la sobrevida de los enfermos, al menos no la empeora. Junto a los betabloqueadores, es el antiarrítmico más conveniente, ya que no tiene efecto inotrópico negativo y su potencial efecto proarrítmico es muy bajo. Cabe insistir que la terapia médica debe estar optimizada con fármacos no antiarrítmicos clásicos como los IECA, betabloqueadores, y antagonistas de la aldosterona, lo cual de por si tiene un rol en la prevención de MS.

La TV sostenida, bien tolerada, en pacientes con FE de VI de $40 \%$ o más puede ser tratada farmacológicamente o mediante ablación con radiofrecuencia. La amiodarona es la droga antiarrítmica actualmente más efectiva disponible para los pacientes que han presentado esta arritmia. La dosis de mantención recomendada es de 200 a $400 \mathrm{mg} / \mathrm{d}$, idealmente asociada a betabloqueadores, si las condiciones lo permiten. La administración de los otros antiarrítmicos antes mencionados, excepto el sotalol en pacientes con buena función ventricular izquierda, aumentan la mortalidad en estos pacientes, por lo que no deben indicarse.

\section{Desfibrilador implantable}

Los pacientes con fibrilación ventricular tardía (después de 48 horas del IAM) y aquellos que presentan TV sostenidas con compromiso hemodinámico o síncope, independiente de la función ventricular y no atribuibles a isquemia, o a otra causa clara corregible, tienen indicación de un desfibrilador automático, única terapia que claramente ha demostrado mejorar la sobrevida en estos pacientes 87,88

Las evidencias actuales señalan que cuando un paciente queda con mala función ventricular post IAM (ya sea $\mathrm{FEVI}<30 \%$ y en CF I, o FEVI $<35 \%$ y en CF II-III), aunque esté asintomático y no se hayan objetivado arritmias ventriculares, el implante profiláctico de un desfibrilador reduce significativamente la muerte súbita y la mortalidad general (comparado con amiodarona y otros antiarrítmicos).

Lo mismo es válido para pacientes con TV no sostenida en un Holter, y en quienes un estudio electrofisiológico induce TV sostenida o FV. Hay también consenso en que los pacientes asintomáticos, con fracción de eyección residual > de $40 \%$ no requieren de desfibrilador, pues tienen bajo riesgo de muerte súbita. Como la función ventricular puede recuperarse (recuperación de la funcionalidad del músculo "atontado"), la decisión del implante de desfibrilador en los pacientes asintomáticos debe postergarse por -al menos- 40 días post IAM89. 
La indicación del implante de un desfibrilador como prevención primaria (aquellos pacientes que no han tenido taquicardia ni fibrilación ventricular), a pesar de las evidencias actuales, es difícil de sostener en países como el nuestro. De ahí que sea necesario intentar separar a pacientes que tienen baja probabilidad de aquellos con mayor probabilidad de presentar muerte súbita. En general, se sabe que aquellos pacientes que quedan con $\mathrm{FE}$ bajo $30 \%$, pero sin otros factores de riesgo, tienen una probabilidad de muerte súbita a 2 años $<5 \%$. Por otro lado, aquellos con $\mathrm{FE}<30 \%$, y aún entre $30 \mathrm{y}$ $40 \% \%$, pero que además tienen bloqueo completo de rama izquierda u otros trastornos de la conducción intraventricular (menos bloqueo de rama derecha), síntomas de insuficiencia cardiaca, TV no sostenida, pobre variabilidad del intervalo $R-R$ y de alternancia de la onda $\mathrm{T}$, potenciales tardíos presentes, historia de fibrilación auricular, y TV inducible en estudio electrofisiológico, tienen mucho mayor riesgo de muerte súbita, y por tanto la indicación de un desfibrilador como prevención primaria está mejor sustentada90. En la Figura 8 se presenta un algoritmo de estratificación de riesgo simplificado y las conductas sugeridas.

Dada la complejidad de una decisión que implica una intervención y un dispositivo de muy alto costo, los pacientes deben ser referidos a centros terciarios. No existe certeza actualmente de cual es la mejor forma de estratificar a estos pacientes. Hay estudios en marcha que pueden ayudar a tomar la decisión del implante profiláctico de un desfibrilador.

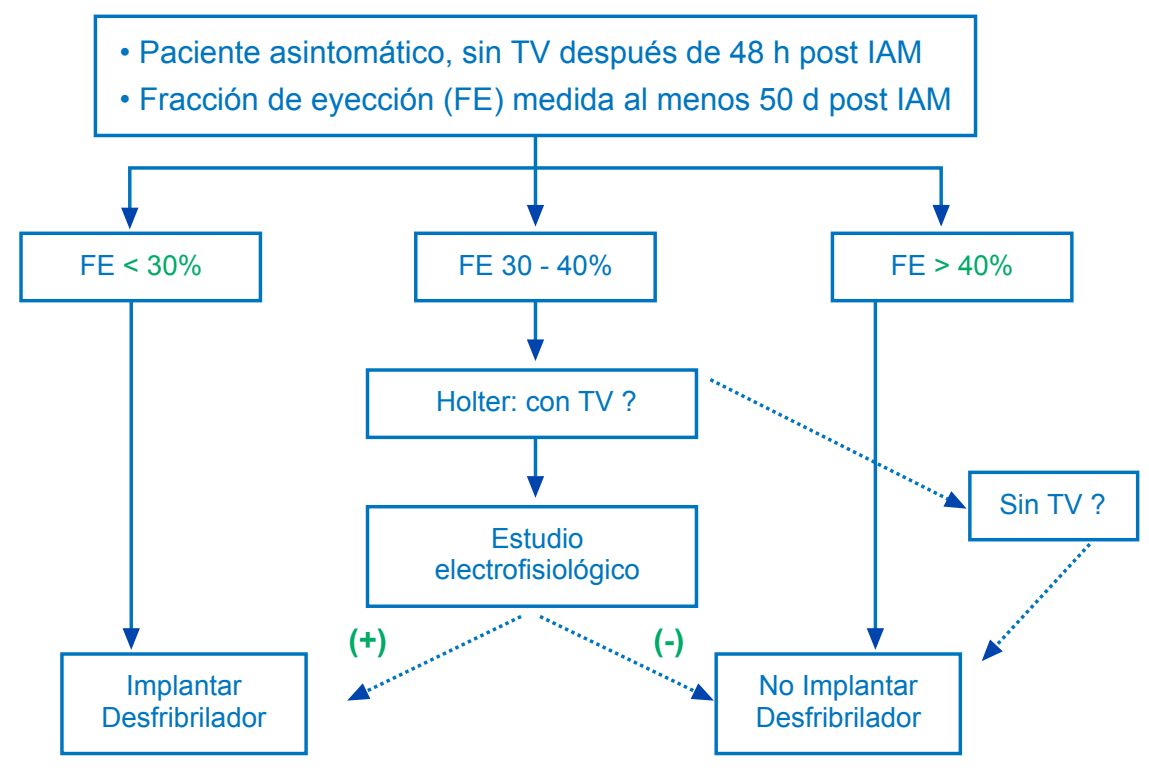

Figura 8: Recomendaciones para implante de Desfibrilador en pacientes con IAM con SDST y baja fracción de eyección

\section{Controles Ambulatorios}

Es muy importante implementar en los centros principales un buen plan de seguimiento de los pacientes egresados tras un IAM con SDST. Gran parte de las complicaciones graves, como reinfarto o muerte súbita podrían evitarse con los medicamentos y exámenes adecuados (tabla 6). Sin embargo, tanto en nuestro país como en el mundo desarrollado, la adherencia a los tratamientos es muy baja, cercana al $70-80 \% 91$. Parte de las razones de la falta de adherencia a los tratamientos son: poca comprensión por parte del paciente y su familia de la importancia de ingerir sus medicamentos ordenadamente, razones económicas para comprarlos, dificultades para conseguir hora en los policlínicos, y también escasa retroalimentación 
positiva de parte del médico. En nuestro país, como en el resto del mundo, el IAM con SDST en mujeres tiene peor pronóstico que en hombres 92 , por lo cual es importante realizar campañas educativas para mejorar la prevención cardiovascular en mujeres. La incorporación de los pacientes a programas de rehabilitación contribuye fuertemente a su recuperación personal y social.

Tabla 6: Objetivos del control ambulatorio post IAM

\begin{tabular}{|l|l|}
\hline Clínicos & Preguntar por recurrencia de síntomas, establecer capacidad funcional \\
\hline Medicamentos & $\begin{array}{l}\text { Chequear uso correcto de los medicamentos. Reevaluar dosis, especialmente de IECA, } \\
\text { Betabloqueadores y de estatinas }\end{array}$ \\
\hline Exámenes & $\begin{array}{l}\text { Continuar con el plan post alta en relación a la FE: revaluar con ecocardiograma a las } 6 \\
\text { semanas, en especial ante FE cercanas al } 40 \% \text {. Eventualmente holter de arritmias para } \\
\text { posible indicación de desfibrilador. } \\
\text { Asegurar control metabólico (glicemia, hemoglobina glicosilada, lípidos plasmáticos) }\end{array}$ \\
\hline Actividad física & $\begin{array}{l}\text { Conversar con el paciente respecto del retorno a la vida normal, incluyendo trabajo, vida } \\
\text { sexual, posibilidad de viajar. Insistir en estilo de vida saludable y ejercicio físico progresivo. } \\
\text { Estimular la abstención del hábito tabáquico }\end{array}$ \\
\hline & $\begin{array}{l}\text { Idealmente incorporar al paciente a un programa de rehabilitación (supervisado), guiado } \\
\text { por test de esfuerzo. Asociar a caminatas, trote, bicicleta. }\end{array}$ \\
\hline
\end{tabular}

\section{Referencias}

1. Antithrombotic Therapy for Non ST-Segment Elevation Acute Coronary Syndromes: American College of Chest Physicians Evidence-Based Clinical Practice Guidelines (8th Edition). American College of Chest Physicians (ACCP). Chest 2008; 133: 670-707.

2. ANTMAN EM, ANBE DT, ARMSTRONG PW, BATES ER, GREEN LA, HAND M, et al. ACC/AHA guidelines for the management of patients with ST-elevation myocardial infarction. J Am Coll Cardiol 2004; 44: E1-E211.

3. ANTMAN EM, HAND M, ARMSTRONG PW, BATES ER, GREEN LA, HALASYAMANI LK, et al. 2007 Focused Update of the ACC/AHA 2004 Guidelines for the Management of Patients With ST-Elevation Myocardial Infarction. Circulation 2008; 117: 296-329.

4. KING SB 3RD, SMITH SC JR, HIRSHFELD JW JR, JACOBS AK, MORRISON DA, WILLIAMS DO, et al. 2007 Focused Update of the ACC/AHA/SCAI 2005 Guideline Update for Percutaneous Coronary Intervention. Circulation 2008; 117: 261-295.

5. Management of acute myocardial infarction in patients presenting with persistent ST - segment elevation. European Society of Cardiology. European Heart Journal 2008; 29: 2909-2945.

6. Ministerio de Salud de Chile. Estudio de carga de enfermedad y carga atribuible, Chile 2007. Documento disponible en www.minsal.cl. Consultado en Julio 2009.

7. CORBALÁN R, NAZZAL C, PRIETO JC, CHÁVEZ E,
LANAS F, LAMICH R, et al. Reducción de la mortalidad por infarto del miocardio en hospitales chilenos. Rev Méd Chile 2002; 130: 368-378

8. TUNSTALL-PEDOE H, KUULASMAA K, MÄHÖNEN M, TOLONEN H, RUOKOKOSKI E, AMOUYEL P. Contribution of trends in survival and coronary-event rates to changes in coronary heart disease mortality: 10-year results from 37 WHO MONICA project populations. Lancet 1999; 353: 1547-1557.

9. GOLDBERG RJ, GLATFELTER K, BURBANK-SCHMIDT E, LESSARD D, GORE JM. Trends in community mortality due to coronary heart disease. Am Heart J 2006; 151: 501-507.

10. Primary versus tenecteplase-facilitated percutaneous coronary intervention in patients with ST-segment elevation acute myocardial infarction (ASSENT-4 PCI): randomised trial. Lancet 2006; 367: 569-578.

11. ALCAYAGA A, GUARDA E, LAZEN R, FLORES A, VALENZUELA E, DEL VALLE F, et al. Retraso entre la consulta y repercusión en pacientes con infarto agudo al miocardio tratados con angioplastía primaria en Santiago. Rev Chil Cardiol 2005; 24: 125-129.

12. GREIG D, CORBALÁN R, CASTRO P, CAMPOS P, LAMICH R, YOVANINIZ P. Impacto de la trombolisis y de la angioplastia primaria en pacientes con infarto agudo del miocardio tratados en centros hospitalarios terciarios. Rev Méd Chile 2008; 136: 1098-1106 
13. PRIETO JC, CORBALÁN R, BARTOLUCCI J, NAZZAL C, CHÁVEZ E, LANAS F, et al, a nombre del Grupo de Estudio Multicéntrico del Infarto (GEMI). Trombolisis en el infarto agudo del miocardio en hospitales chilenos. La experiencia del grupo GEMI. Rev Chil Cardiol 2003; 22: 23-30.

14. PRIETO JC, SANHUEZA C, MARTINEZ N, NAZZAL C, CORBALÁN R, CAVADA G, et al. a nombre de investigadores del Grupo de Estudio Multicéntrico del Infarto (GEMI). Mortalidad intrahospitalaria en hombres y mujeres según terapias de reperfusión en infarto agudo del miocardio con supradesnivel del ST. Rev Med Chil. 2008; 136:143-150.

15. DE LUCA G, SURYAPRANATA H, OTTERVANGER JP, ANTMAN EM. Time delay to treatment and mortality in primary angioplasty for acute myocardial infarction. Circulation 2004; 109: 1223-1225.

16. ANDERSON JL, ADAMS CD, ANTMAN EM, BRIDGES CR, CALIFF RM, CASEY DE, et al. ACC/AHA 2007 Guidelines for the Management of Patients With Unstable Angina/Non-ST-Elevation Myocardial Infarction. J Am Coll Cardiol 2007; 50:1-157.

17. BASSAND JP, HAMM CW, ARDISSINO D, BOERSMA E, BUDAJ A, FERNÁNDEZ-AVILÉS $F$, et al. Guidelines for the diagnosis and treatment of non-ST-segment elevation acute coronary syndromes. Eur Heart J 2007; 28: $1598-660$.

18. MORRISON LJ, VERBEEK PR, MCDONALD AC, SAWADSKY BV, COOK DJ. Mortality and prehospital thrombolysis for acute myocardial infarction: A meta-analysis. JAMA 2000; 283: 2686-92.

19. BJÖRKLUND E, STENESTRAND U, LINDBÄCK J, SVENSSON L, WALLENTIN L, LINDAHL B. Pre-hospital thrombolysis delivered by paramedics is associated with reduced time delay and mortality in ambulancetransported real-life patients with ST-elevation myocardial infarction. Eur Heart J 2006; 27: 1146-1152.

20. DANCHIN N, BLANCHARD D, STEG PG, SAUVAL P, HANANIA G, GOLDSTEIN P, et al. Impact of prehospital thrombolysis for acute myocardial infarction on 1-year outcome: results from the French nationwide USIC 2000 registry. Circulation 2004; 110: 1909-1915.

21. VAN'T HOF AW, TEN BERG J, HEESTERMANS T, DILL T, FUNCK RC, VAN WERKUM $W$, et al. Prehospital initiation of tirofiban in patients with ST-elevation myocardial infarction undergoing primary angioplasty (On-TIME 2): a multicentre, double-blind, randomised controlled trial. Lancet 2008; 372: 537-46

22. WIDIMSKY P, BUDESINSKY T, VORAC D, GROCH L, ZELIZKO M, ASCHERMANN M, BRANNY M, et al. Long distance transport for primary angioplasty vs immediate thrombolysis in acute myocardial infarction: final results of the randomized national multicentre trial: PRAGUE-2. Eur Heart J 2003; 24: 94-104.

23. ANTMAN EM, BENNETT JS, DAUGHERTY A, FURBERG C, ROBERTS H, TAUBERT KA; American Heart Association. Use of nonsteroidal antiinflammatory drugs: an update for clinicians: a scientific statement from the American Heart Association. Circulation 2007; 115: 1634-42.

24. GISLASON GH, JACOBSEN S, RASMUSSEN JN, RASMUSSEN S, BUCH P, FRIBERG J, et al. Risk of death or reinfarction associated with the use of selective cyclooxygenase-2 inhibitors and nonselective nonsteroidal antiinflammatory drugs after acute myocardial infarction. Circulation 2006; 113: 2906-13.

25. CHEN ZM, PAN HC, CHEN YP, PETO R, COLLINS R, JIANG LX, et al. Early intravenous then oral metoprolol in 45,852 patients with acute myocardial infarction: randomised placebo-controlled trial (COMMIT TRIAL). Lancet 2005; 366: 1622-1632.

26. FREEMANTLE N, CLELAND J, YOUNG P, MASON J, HARRISON J. Beta blockade after myocardial infarction: systematic review and meta regression analysis. BMJ 1999; 318: 1730-1737.

27. CHEN ZM, JIANG LX, CHEN YP, XIE JX, PAN HC, PETO $R$, et al. Addition of clopidogrel to aspirin in 45,852 patients with acute myocardial infarction: randomised placebo-controlled trial. Lancet 2005; 366: 1607-21.

28. SABATINE MS, CANNON CP, GIBSON CM, LÓPEZSENDÓN JL, MONTALESCOT G, THEROUX P, et al. Addition of clopidogrel to aspirin and fibrinolytic therapy for myocardial infarction with STsegment elevation. N Engl J Med 2005; 352: 1179-89.

29. VON BECKERATH N, TAUBERT D, POGATSA-MURRAY G, SCHÖMIG E, KASTRATI A, SCHÖMIG A. Absorption, metabolization, and antiplatelet effects of 300-, 600-, and 900-mg loading doses of clopidogrel: results of the ISAR-CHOICE Trial. Circulation 2005; 112: 2946-50.

30. A randomised factorial trial assessing early oral captopril, oral mononitrate, and intravenous magnesium in 58,050 patients with suspected acute myocardial infarction. ISIS-4. Lancet 1995; 345: 669-685.

31. YUSUF S, HELD P, FURBERG C. Update of effects of calcium antagonists in myocardial infarction or angina in light of the second Danish Verapamil Infarction Trial (DAVIT-II) and other recent studies. Am J Cardiol 1991; 67: 1295-1297.

32. JOSAN K, MAJUMDAR SR, MCALISTER FA. The efficacy and safety of intensive statin therapy: a 
meta-analysis of randomized trials. CMAJ 2008; 178 : 576-84

33. KEELEY EC, BOURA JA, GRINES CL. Primary angioplasty versus intravenous thrombolytic therapy for acute myocardial infarction: a quantitative review of 23 randomised trials. Lancet 2003; 361: 13-20

34. GUARDA E, MIRANDA R, MARTÍNEZ A, FAJURI A, MARCHANT E, LAZEN R, et al. Angioplastía primaria como estrategia de tratamiento del IAM con SDST. Rev Chil Cardiol 2006; 25; 122-123.

35. MARTINEZ A, NAZZAL C, FAJURI A, y cols, en representacion de los registros RENAC y GEMI. Mortalidad post infarto del miocardio en Chile: Comparación de los registros de angioplastía primaria versus trombolisis. Aceptado para publicación en Rev Méd Chile 2009

36. NAZZAL C, CAMPOS C, CORBALÁN R, LANAS F, BARTOLUCCI J, SANHUEZA P, et al. Impacto del plan AUGE en el tratamiento de pacientes con infarto agudo del miocardio con supradesnivel del ST en hospitales chilenos. Rev Med Chil 2008; 136:1231-1239.

37. PÉREZ L, LECANNELIER E, OLMOS A, SALDAÑA A, VENEGAS R, PARRA J, et al. Angioplastía primaria en el infarto agudo al miocardio en el Hospital Regional de Concepción. Rev Chil Cardiol 2007; 26: 391-397

38. BRADLEY EH, HERRIN J, WANG Y, BARTON BA, WEBSTER TR, MATTERA JA, et al. Strategies for reducing the door-to-balloon time in acute myocardial infarction. N Engl J Med. 2006: 355: 2308-2320.

39. DALBY M, BOUZAMONDO A, LECHAT P, MONTALESCOT G. Transfer for primary angioplasty versus immediate thrombolysis in acute myocardial infarction: a meta-analysis. Circulation 2003; 108: 1809-14.

40. HENRY TD, UNGER BT, SHARKEY SW, LIPS DL, PEDERSEN WR, MADISON JD, et al. Design of a standardized system for transfer of patients with ST-elevation myocardial infarction for percutaneous coronary intervention. Am Heart J 2005; 150: 373-84.

41. A clinical trial comparing primary coronary angioplasty with tissue plasminogen activator for acute myocardial infarction. (GUSTO Ilb Angioplasty Substudy Investigators). N Engl J Med 1997; 336: 1621-1628

42. ANDERSEN HR, NIELSEN TT, RASMUSSEN K, THUESEN L, KELBAEK H, THAYSSEN $P$, et al. A comparison of coronary angioplasty with fibrinolytic therapy in acute myocardial infarction. $\mathrm{N}$ Engl J Med 2003; 349: 733-742.

43. LEÓN L, GUARDA E, GABRIELLI L, MIRANDA R, PÉREZ O, MÉNDEZ M, et al. Resultados Clínicos y Angiográficos de la Trombolisis en el Hospital Sótero del Río. Rev Chil Cardiol 2007; 26: 23-27.

44. BODEN WE, EAGLE K, GRANGER CB. Reperfusion Strategies in Acute ST-Segment Elevation Myocardial Infarction: A Comprehensive Review of Contemporary Management Options. J Am Coll Cardiol 2007; 50: 917-929.

45. NALLAMOTHU BK, WANG Y, MAGID DJ, MCNAMARA RL, HERRIN J, BRADLEY EH, et al. Relation between hospital specialization with primary percutaneous coronary intervention and clinical outcomes in

ST-segment elevation myocardial infarction: National Registry of Myocardial Infarction-4 analysis. Circulation 2006; 113: 222-9.

46. SAIA F, MARROZZINI C, ORTOLANI P, PALMERINI T, GUASTAROBA P, CORTESI P, et al. Optimisation of therapeutic strategies for ST-segment elevation acute myocardial infarction: the impact of a territorial network on reperfusion therapy and mortality. Heart 2009; 95:70-6.

47. DE LUCA G, SURYAPRANATA H, STONE GW, ANTONIUCCI D, TCHENG JE, NEUMANN FJ, et al. Abciximab as adjunctive therapy to reperfusion in acute ST-segment elevation myocardial infarction: a meta-analysis of randomized trials. JAMA 2005; 293: 1759-1765.

48. CUTLIP DE, RICCIARDI MJ, LING FS, CARROZZA JP JR, DUA V, GARRINGER J, et al. Effect of tirofiban before primary angioplasty on initial coronary flow and early ST-segment resolution in patients with acute myocardial infarction. Am J Cardiol 2003; 92: 977-80.

49. GYONGYOSI M, DOMANOVITS $H$, BENZER $W$, HAUGK M, HEINISCH B, SODECK G, et al. Use of abciximab prior to primary angioplasty in STEMI results in early recanalization of the infarct-related artery and improved myocardial tissue reperfusion-results of the Austrian multi-centre randomized ReoPro-BRIDGING Study. Eur Heart J 2004; 25: 2125-33.

50. Primary versus tenecteplase-facilitated percutaneous coronary intervention in patients with ST-segment elevation acute myocardial infarction (ASSENT-4 PCI): randomised trial. Lancet 2006; 367: 569-78.

51. WIJEYSUNDERA HC, VIJAYARAGHAVAN R, NALLAMOTHU BK, FOODY JM, KRUMHOLZ HM, PHILLIPS CO, et al. Rescue angioplasty or repeat fibrinolysis after failed fibrinolytic therapy for ST-segment myocardial infarction: a meta-analysis of randomized trials. J Am Coll Cardiol 2007; 49: 422-430.

52. HOCHMAN JS, SLEEPER LA, WEBB JG, SANBORN TA, WHITE HD, TALLEY JD, et al. Early revascularization 
in acute myocardial infarction complicated by cardiogenic shock. SHOCK Investigators. N Engl J Med 1999; 341: 625-634.

53. Indications for fibrinolytic therapy in suspected acute myocardial infarction: collaborative overview of early mortality and major morbidity results from all randomised trials of more than 1000 patients. Fibrinolytic Therapy Trialists' (FTT) Collaborative Group. Lancet 1994; 343: 311-322.

54. WHITE H. Thrombolytic therapy in the elderly. Lancet 2000; 356: 2028-2030.

55. BOERSMA H, MAAS AC, DECKERS JW, SIMOONS ML. Early thrombolytic treatment in acute myocardial infarction: reappraisal of the golden hour. Lancet 1996; 348: 771-775.

56. VAN DE WERF F, ADGEY J, ARDISSINO D, ARMSTRONG PW, AYLWARD P, BARBASH G, et al. Single-bolus tenecteplase compared with front-loaded alteplase in acute myocardial infarction: the ASSENT-2 double-blind randomised trial. Lancet 1999; 354: 716-722.

57. Efficacy and safety of tenecteplase in combination with enoxaparin, abciximab, or unfractionated heparin: the ASSENT-3 randomised trial in acute myocardial infarction. Lancet 2001; 358: 605-613.

58. PETERS RJ, JOYNER C, BASSAND JP, AFZAL R, CHROLAVICIUS S, MEHTA SR, et al. The role of fondaparinux as an adjunct to thrombolytic therapy in acute myocardial infarction: a subgroup analysis of the OASIS-6 trial. Eur Heart J 2008; 29: 324-331.

59. ARMSTRONG PW, BOGATY P, BULLER CE, DORIAN P, O'NEILL BJ. The 2004 ACC/AHA Guidelines: a perspective and adaptation for Canada by the Canadian Cardiovascular Society Working Group. Can J Cardiol 2004; 20: 1075-9.

60. GORE JM, GRANGER CB, SIMOONS ML, SLOAN MA, WEAVER WD, WHITE HD, et al. Stroke after thrombolysis. Mortality and functional outcomes in the GUSTO-I trial. Circulation 1995; 92: 2811-2818.

61. RAFFO C, BARTOLUCCI J, CORBALÁN R, ANINAT M, TORRES H, PRIETO JC, et al. Utilidad de la terapia trombolítica con estreptoquinasa a dosis baja en infarto agudo del miocárdio. Revista Med Chil 2006; 134: 1249-1257.

62. SCHOMIG A, MEHILLI J, ANTONIUCCI D, NDREPEPA G, MARKWARDT C, DI PEDE F, et al. Mechanical reperfusion in patients with acute myocardial infarction presenting more than 12 hours from symptom onset: a randomized controlled trial. JAMA 2005; 293: 2865-2872.

63. LEE DC, OZ MC, WEINBERG AD, TING W. Appropriate timing of surgical intervention after transmural acute myocardial infarction. J Thorac Cardiovasc Surg 2003; 125: 115-119.

64. BRODIE BR, STUCKEY TD, HANSEN C, BRADSHAW
BH, DOWNEY WE, PULSIPHER MW. Comparison of late survival in patients with cardiogenic shock due to right ventricular infarction versus left ventricular pump failure following primary percutaneous coronary intervention for ST-elevation acute myocardial infarction. Am J Cardiol 2007; 99: 431-435.

65. HJALMARSON A. Effects of beta blockade on sudden cardiac death during acute myocardial infarction and the postinfarction period. Am J Cardiol 1997; 80: 35J-39J.

66. ZIPES DP, CAMM AJ, BORGGREFE M, BUXTON AE, CHAITMAN B, FROMER $M$, et al. ACC/AHA/ESC 2006 guidelines for management of patients with ventricular arrhythmias and the prevention of sudden cardiac death--executive summary. Eur Heart J 2006; 27: 2099-2140.

67. RATHORE SS, BERGER AK, WEINFURT KP, SCHULMAN KA, OETGEN WJ, GERSH BJ, et al. Acute myocardial infarction complicated by atrial fibrillation in the elderly: prevalence and outcomes. Circulation 2000; 101: 969-974.

68. FUSTER V, RYDEN LE, CANNOM DS, CRIJNS HJ, CURTIS AB, ELLENBOGEN KA, et al. ACC/AHA/ESC 2006 Guidelines for the Management of Patients with Atrial Fibrillation: a report of the American College of Cardiology/American Heart Association Task Force on Practice Guidelines and the European Society of Cardiology Committee for Practice Guidelines. Circulation 2006; 114: e257-e354.

69. NEWBY KH, PISANO E, KRUCOFF MW, GREEN C, NATALE $A$. Incidence and clinical relevance of the occurrence of bundle-branch block in patients treated with thrombolytic therapy. Circulation 1996; 94: 2424-2428.

70. GREGORATOS G, ABRAMS J, EPSTEIN AE, FREEDMAN RA, HAYES DL, HLATKY MA, et al. ACC/AHA/NASPE 2002 guideline update for implantation of cardiac pacemakers and antiarrhythmia devices: summary article. Circulation 2002; 106: 2145-2161.

71. TOPAZ O, TAYLOR AL. Interventricular septal rupture complicating acute myocardial infarction: from pathophysiologic features to the role of invasive and noninvasive diagnostic modalities in current management. Am J Med 1992; 93: 683-688.

72. CHEVALIER P, BURRI H, FAHRAT F, CUCHERAT M, JEGADEN O, OBADIA JF, et al. Perioperative outcome and long-term survival of surgery for acute post-infarction mitral regurgitation. Eur J Cardiothorac Surg 2004; 26 : 330-335.

73. CHEN Q, DARLYMPLE-HAY MJ, ALEXIOU C, OHRI SK, HAW MP, LIVESEY SA, et al. Mitral valve surgery for 
acute papillary muscle rupture following myocardial infarction. Heart Valve Dis 2002; 11: 27-31.

74. DICKSTEIN K, COHEN-SOLAL A, FILIPPATOS G, MCMURRAY JJV, PONIKOWSKI P, POOLE-WILSON $P A$, et al. ESC Guidelines for the diagnosis and treatment of acute and chronic heart failure 2008. Eur Heart J 2008; 29: 2388-2442.

75. HOCHMAN JS, SLEEPER LA, WHITE HD, DZAVIK $\mathrm{V}$, WONG SC, MENON $\mathrm{V}$, et al. One-year survival following early revascularization for cardiogenic shock. JAMA 2001; 285: 190-192.

76. GRAHAM I, ATAR D, BORCH-JOHNSEN K, BOYSEN G, BURELL G, CIFKOVA R, et al. European guidelines on cardiovascular disease prevention in clinical practice: executive summary. Eur Heart J 2007; 28: 2375-2414.

77. SMITH SC JR, ALLEN J, BLAIR SN, BONOW RO, BRASS LM, FONAROW GC, et al. AHA/ACC guidelines for secondary prevention for patients with coronary and other atherosclerotic vascular disease: 2006 update: endorsed by the National Heart, Lung, and Blood Institute. J Am Coll Cardiol 2006; 47: 2130-9.

78. MANCIA G, DE BACKER G, DOMINICZAK A, CIFKOVA R, FAGARD R, GERMANO $G$, et al. The task force for the management of arterial hypertension of the European Society of Hypertension. Eur Heart J 2007; 28: 1462-1536.

79. REUS VI, SMITH BJ. Multimodal techniques for smoking cessation: a review of their efficacy and utilisation and clinical practice guidelines. Int $\mathrm{J}$ Clin Pract 2008; 62: 1753-68.

80. YUSUF S, HELD P, FURBERG C. Update of effects of calcium antagonists in myocardial infarction or angina in light of the second Danish Verapamil Infarction Trial (DAVIT-II) and other recent studies. Am J Cardiol 1991; 67: 1295-1297.

81. GISSI-3. Effects of lisinopril and transdermal glyceryl trinitrate singly and together on 6-week mortality and ventricular function after acute myocardial infarction. Lancet 1994; 343: 1115-1122.

82. ISIS-4. A randomised factorial trial assessing early oral captopril, oral mononitrate, and intravenous magnesium in 58,050 patients with suspected acute myocardial infarction. Lancet 1995; 345: 669-685.

83. DANCHIN N, CUCHERAT M, THUILLEZ C, DURAND E, KADRI Z, STEG PG. Angiotensin-converting enzyme inhibitors in patients with coronary artery disease and absence of heart failure or left ventricular systolic dysfunction: an overview of long-term randomized controlled trials. Arch Intern Med 2006; 166: 787-796.

84. DICKSTEIN K, KJEKSHUS J. Effects of losartan and captopril on mortality and morbidity in high-risk patients after acute myocardial infarction: the OPTIMAAL randomised trial. Lancet 2002; 360: 752-760.

85. PFEFFER MA, MCMURRAY JJ, VELAZQUEZ EJ, ROULEAU JL, KOBER L, MAGGIONI AP, et al. Valsartan, captopril, or both in myocardial infarction complicated by heart failure, left ventricular dysfunction, or both. $\mathrm{N}$ Engl J Med 2003; 349: 1893-1906.

86. PITT B, REMME W, ZANNAD F, NEATON J, MARTINEZ $F$, RONIKER B, et al. Eplerenone, a selective aldosterone blocker, in patients with left ventricular dysfunction after myocardial infarction. N Engl J Med 2003; 348: 1309-1321.

87. EZEKOWITZ JA, ARMSTRONG PW, MCALISTER FA. Implantable cardioverter defibrillators in primary and secondary prevention: a systematic review of randomized, controlled trials. Ann Intern Med 2003; 138: 445-52.

88. BARDY GH, LEE KL, MARK DB, POOLE JE, PACKER $\mathrm{DL}$, BOINEAU R, et al. Amiodarone or an implantable cardioverter-defibrillator for congestive heart failure. N Engl J Med 2005; 352: 225-37.

89. BUXTON AE, LEE KL, HAFLEY GE, PIRES LA, FISHER JD, GOLD MR, et al. Limitations of ejection fraction for prediction of sudden death risk in patients with coronary artery disease: lessons from the MUSTT study. J Am Coll Cardiol 2007; 50: 1150-7.

90. MOSS AJ, ZAREBA W, HALL WJ, KLEIN H, WILBER DJ, CANNOM DS, et al. Prophylactic implantation of a defibrillator in patients with myocardial infarction and reduced ejection fraction. N Engl J Med 2002; 346: 877-883.

91. GUARDA E, ACEVEDO M, LIRA MT, CHAMORRO G, CORBALÁN R. Prevalence of cardiovascular risk factors among patients suffering vascular events on admission and one year later. Rev Med Chil 2005; 133: 1147-52.

92. MARTÍNEZ A, MARCHANT E, GUARDA E, FAJURI A, DUSSAILLANT G, PÉRES $O$, et al. Influencia del Género en la Mortalidad del Infarto del Miocardio tratado con Angioplastía Primaria. Rev Chil Cardiol 2005; 24: 71-76. 